\title{
Polyhedral Clinching Auctions and the Adwords Polytope
}

\author{
Gagan Goel \\ Google Inc., New York \\ gagangoel@google.com
}

\author{
Vahab Mirrokni \\ Google Inc., New York \\ mirrokni@google.com
}

\author{
Renato Paes Leme* \\ Cornell University \\ renatoppl@cs. cornell. edu
}

May 29, 2018

\begin{abstract}
A central issue in applying auction theory in practice is the problem of dealing with budgetconstrained agents. A desirable goal in practice is to design incentive compatible, individually rational, and Pareto optimal auctions while respecting the budget constraints. Achieving this goal is particularly challenging in the presence of nontrivial combinatorial constraints over the set of feasible allocations.

Toward this goal and motivated by AdWords auctions, we present an auction for polymatroidal environments satisfying the above properties. Our auction employs a novel clinching technique with a clean geometric description and only needs an oracle access to the submodular function defining the polymatroid. As a result, this auction not only simplifies and generalizes all previous results, it applies to several new applications including AdWords Auctions, bandwidth markets, and video on demand. In particular, our characterization of the AdWords auction as polymatroidal constraints might be of independent interest. This allows us to design the first mechanism for Ad Auctions taking into account simultaneously budgets, multiple keywords and multiple slots.

We show that it is impossible to extend this result to generic polyhedral constraints. This also implies an impossibility result for multi-unit auctions with decreasing marginal utilities in the presence of budget constraints.
\end{abstract}

\footnotetext{
*This author's contribution to this work was made while he was an intern at Google Inc. He is currently a graduate student at Cornell University supported by a Microsoft Fellowship.
} 


\section{Introduction}

A large part of auction theory deals with the problem of designing truthful mechanisms for quasilinear settings. For these settings, the VCG mechanism, or variants of it like affine-maximizers, can be applied to get optimal or near-optimal auctions. However, when we deviate from the quasi-linear model, very little is known. One of the most natural and practically important feature missing from the quasi-linear model is the presence of budget constraints. Budgets play a major role in several real-world auctions where the magnitude of the transactions involved naturally put a financial constraint on the bidders. Examples of such auctions include those used for the privatization of public assets in eastern Europe, or those for the distribution of radio spectra in the US (for a discussion on this, see Benoit and Krishna [6]). Another important example is that of Ad Auctions where advertisers explicitly declare budget constraints. In such settings, respecting the declared budget constraints is a necessary property any mechanism must satisfy. There is much discussion on the source of budget constraints (we refer to Che and Gale [11] for a detailed discussion on this topic).

Satisfying budget constraints while keeping incentive compatibility and efficiency is a challenging problem, and it becomes even harder in the presence of complex combinatorial constraints over the set of feasible allocations. In the presence of budgets, individual rationality and truthfulness cannot be satisfied at the same time as maximizing social welfare [14], and thus the goal of maximizing efficiency can be achieved mainly through Pareto-optimal auctions 1 . Therefore, a desirable goal under budget constraints is to design incentive-compatible (IC) and individually-rational (IR) auctions while producing Pareto-optimal outcomes. The first successful example of such mechanisms was developed in the seminal paper of Dobzinski, Lavi and Nisan [14], where the authors adapt the clinching auction framework of Ausubel [5] to give a truthful mechanism that achieves Pareto-optimality. Their setting, however, captures only a simple allocation constraint: there is a limited supply of $k$ items and each player has a value of $v_{i}$ for each item (and hence value of $v_{i} \cdot t$ for getting $t$ items) and budget $B_{i}$.

As for more general allocation constraints, there have been a couple of subsequent work capturing special families of allocation constraints, e.g., unit demands [2], or multi-unit demands with matching constraints [18]. Although these results are mainly based on Ausubel's clinching auction, each of them need to develop independent techniques to deal with their specific environment - for example, Fiat, Leonardi, Saia, and Sankowski [18] define a complex clinching procedure based on trading paths in a bipartite graph and computing $S$-avoid matchings. Our goal in this paper is to extend these results to a much more general class of polyhedral constraints. In particular, we would like to understand for what polyhedral environments one can design such auctions, and also identify simple environments for which designing such auctions is not possible.

Our Results and Techniques. Firstly, inspired by an application in Sponsored Search Ad Auctions and several other applications, we study polymatroid constraints over feasible allocations and give an auction that achieves all the desired properties, i.e., it satisfies IC, IR, and produces Pareto-optimal outcomes while satisfying the budget constraints. We assume that the budgets are public - which was shown in [14] to be a necessary assumption 2]. While following Ausubel's framework to design this auction, we need to invent the main component of the mechanism, i.e.,

\footnotetext{
${ }^{1}$ An auction is Pareto-optimal if it outputs an allocation and payments such that no alternative set of allocation and payments improves the utility of at least one agent and keeps the other agents at least as happy as before. Here agents include bidders and the auctioneer where the auctioneer's utility is its revenue

${ }^{2}$ Dobzinski et. al. 14] showed that with private budgets, truthfulness and Pareto-optimality cannot be achieved using deterministic mechanisms - not even for multi-unit auctions
} 
the clinching step that copes with the polyhedral allocation constraints. Our clinching step uses submodular minimization as a subroutine and only needs a value oracle access to the submodular function corresponding to the polymatroid. As a result, our mechanism has a clean geometric description that abstracts away the combinatorial complications of previous designs. This leaves the auctioneer free to focus on modeling the environment, and then use our mechanism as a blackbox. This general technique not only generalizes (and simplifies) the previously known results like multi-unit auctions with matching constraints [14, 18, but also extend clinching auctions to many other applications like the AdWords Auction and settings like spanning tree auctions and video on demand [8]. Our main application is in sponsored search auctions where we model the AdWords Auction with multiple keywords and multiple position slots per keyword as a polymatroid called the AdWords polytope (See Section 4.1 for details).

In order to extend this result to more general polyhedral constraints, we turn our attention to 2-player auctions with budget constraints and prove several structural properties of Pareto-optimal truthful auctions for polyhedral environments In particular, we present a characterization of such auctions that results in various impossibility results and one positive result. On the positive side, we present a truthful individually rational Pareto-optimal auction for any environment if only one player is budget-constrained. On the other hand, if more than one player is budget-constrained, we illustrate simple polytope constraints for which it is impossible to achieve a truthful Paretooptimal auction even for two players. Moreover, as a byproduct of this characterization, we get an impossibility result for multi-unit auctions with decreasing marginal utilities. This impossibility result disproves an implied conjecture by Ausubel [5] which has been reinforced by follow-up papers [14, 21]. In fact, this conjecture was reinforced by the fact that getting such an auction is possible whenever the marginals are flat. In appendix $\mathrm{D}$ we provide an explicit counter-example for this case.

Applications to Sponsored Search. Online advertisement is a growing business that was worth 25 billion dollars in 2010. It also has become a central piece in the current internet landscape, since it is the primary way internet companies monetize their services. Large part of this revenue comes from the search advertisement, hence it is not surprising that is has been extensively studied in the literature. The basic model of sponsored search ad auctions was proposed simultaneously by Edelman, Ostrovky and Schwarz [16] and Varian [31]. The authors model the current auction as a non-truthful mechanism and analyze its equilibrium properties. The social welfare of such equilibria were studied in [28, 22, 10] and its revenue properties in [23].

In search advertising, there are usually multiple keywords and each keyword has multiple slots associated with it. Most of the previous work treat auctions for different keywords as being independent, and therefore focus on a single keyword. A recent paper by Dhangwatnotai [13] approaches the problem of analyzing keyword auctions for multiple keywords, but it is restricted to a special case of one slot per page. Similarly, the work of Fiat et al [18] can be seen as an auction for multiple keywords with only one slot per page.

To the best of our knowledge, our work is the first work to combine multiple slots per page and multiple keywords. We do so by giving a non-trivial characterization of the set of all feasible allocations of clicks: we call it the AdWords Polytope. We show it has the structure of a polymatroid and therefore we can use our result to generate a truthful Pareto-optimal auction for this setting.

Other Related Work. There are two streams of related work. The first, like ours, is on designing truthful mechanisms when players have budget constraints with the goal of achieving Paretooptimal outcomes; for example, [14], and [18]. Bhattacharya et al [7] show a budget-monotonicity property for the clinching auction of [14], therefore arguing that no player can improve his utility by under-reporting his budget. For the case of unit-demand players, Aggrawal et al [2] design auctions 
for unit-demand players with budget constraints.

On the question of maximizing revenue, Borgs et al [9] gave a truthful auction whose revenue is asymptotically within a constant factor of the optimal revenue. These results were improved by Abrams [1]. Subsequently, Hafalir, Ravi and Sayedi [20] relax the truthfulness requirement, moving to ex-post Nash equilibrium as a solution concept, and give an auction that, in equilibrium, has good efficiency and revenue properties. More recently, Pai and Vohra 29] gave a revenue-optimal auction for the Bayesian version of the problem. We would like to highlight that the above work focused on the multi-unit setting only.

Our auction also generalizes the ascending auction of Bikhchandani et al [8]. The authors consider environments where the set of allocations is defined by a polymatroid, but don't consider budget constraints.

The second line of related work relates to the practical problem of designing mechanisms for Ad Auctions. The work of Feldman et al [17] design an auction for the environment with one keyword and multiple slots. Their model is, however, different from the standard utilitarian utility model. Instead of being profit maximizers, the players are clicks maximizers, i.e., the players want to get as many clicks as possible without exhausting their budget and without paying more per click than their value, which is a simpler setting than ours. In order to design their auction, they describe the structure of the set of possible randomized allocations of players to slots. We note that the structure they identify is in fact a polymatroid and use this fact to apply our auction to this setting. We further extend this characterization to the setting with multiple keywords.

Also for one keyword and multiple slots, Ashlagi et al [4] design an auction for the usual utility model but relax the truthfulness requirement and get an auction that is Pareto-optimal for all ex-post Nash equilibria. The main weakness in the setting of [4] is that the agents are allowed to be allocated only to one slot position for all the different queries of the given keyword. However, in reality, agents can be allocated to different slot positions for different queries of a given keyword. In the restricted setting of 4, the Pareto-optimality requirement becomes easier to satisfy.

Independently of our work, Colini-Baldeschi et al [12 also study the problem of designing incentive compatible, individually rational, budget feasible and Pareto-optimal auctions for sponsored search. The authors present two auctions satisfying those properties: one for the case with a single keyword but multiple slots with different click-through-rates and one for the case of multiple keywords and multiple slots with homogeneous click-through-rates (i.e. all slots are identical).

Impossibility results. The impossibility of a Pareto-optimal auction for heterogeneous goods in the budgeted setting was given in [18. It remained an open problem whether an auction was possible if goods where identical, i.e., utilities depended only on the number items acquired and not on which items they were. A very recent result by Lavi and May [21] shows an impossibility result for the case where the valuation can be an arbitrary function of the number of items - i.e. players are allowed to express complementarities. Since their setting is more expressive, an impossibility result is easier. Our impossibility result for multi-unit auctions can be seen as a stronger version of their result, since we allow players only to express valuations with diminishing marginals. This came as a surprise to us, since it was generally believed that such a positive result could be achieved using a variation of [14]. 


\section{Auctions for Polyhedral Environments}

Consider $n$ players, where player $i$ has a positive value $v_{i}$ per unit of some good $g$ and a budget of $B_{i}$. We assume that the valuations are private information of the players, whereas the budgets are public. We are also given a subset $X \subseteq \mathbb{R}_{+}^{n}$ that defines all the possible ways to allocate the good $g$. We assume that the subset $X$ is a convex set that is bounded and downward closed 3 . We will call this set $X$ an environment. Note that if player $i$ receives $x_{i}$ amount of good $g$ and pays $p_{i}$, her utility $u_{i}$ is equal to $v_{i} x_{i}-p_{i}$ if $p_{i} \leq B_{i}$ and $-\infty$ otherwise. However, since we will require the mechanism to never charge more than the budgets, we won't have to deal with the latter case. Our goal is to design an auction mechanism that elicits valuations $v$ from the players and outputs a feasible allocation $x(v) \in X$ and a feasible payment vector $p(v) \leq B$ that satisfies the following three properties:

- Individual Rationality (a.k.a. voluntary participation): Each player has net non-negative utility from participating in the auction, i.e., $u_{i} \geq 0$.

- Incentive compatibility (a.k.a. truthfulness) : It is a dominant strategy for each player to participate in the auction and report their true value, i.e., $v_{i} x_{i}\left(v_{i}, v_{-i}\right)-p_{i}\left(v_{i}, v_{-i}\right) \geq$ $v_{i} x_{i}\left(v_{i}^{\prime}, v_{-i}\right)-p_{i}\left(v_{i}^{\prime}, v_{-i}\right)$. The characterization of single-parameter truthful mechanisms in [25, 3] states that this is equivalent to $x_{i}$ being a non-decreasing function of $v_{i}$ (for a fixed $\left.v_{-i}\right)$ and payments being calculated by $p_{i}\left(v_{i}, v_{-i}\right)=v_{i} x_{i}\left(v_{i}, v_{-i}\right)-\int_{0}^{v_{i}} x_{i}\left(u, v_{-i}\right) d u$.

- Pareto-optimality: An allocation $x(v) \in X$ and payments $p(v) \leq B$ is Pareto-optimal if and only if there is no alternative allocation and payments where all players' utilities and the revenue of the auctioneer do not decrease, and at least one of them increases. In other words, there is no alternative $\left(x^{\prime}, p^{\prime}\right)$ such that $v_{i} x_{i}^{\prime}-p_{i}^{\prime} \geq v_{i} x_{i}(v)-p_{i}(v), \sum_{i} p_{i}^{\prime} \geq \sum_{i} p_{i}(v)$ and at least one of those inequalities is strict.

Next we prove a useful lemma about the structure of Pareto-optimal outcomes.

Lemma 2.1 A feasible outcome $(x, p)$, i.e. $x \in X$ and $p \leq B$, is Pareto-optimal iff there is no $d \in \mathbb{R}^{n}$ in a dominated direction at $x$ (i.e. $x+d \in X^{0}:=\left\{x^{\prime} \in X ; \exists \hat{x} \in X \backslash x^{\prime}, \hat{x} \geq x^{\prime}\right\}$ ) such that $d^{t} v \geq 0$ and $d_{i} \leq 0$ for all $i$ that have $p_{i}=B_{i}$.

Proof : In order to show the $\Rightarrow$ direction, assume there is a dominated direction $d$ such that $d^{t} v \geq 0$ and $d_{i} \leq 0$ for all $i$ that have $p_{i}=B_{i}$. Then define $x_{i}^{\prime}=x_{i}+d_{i}$ and $p_{i}^{\prime}=p_{i}+v_{i} d_{i}$ and we obtain same utilities and the total payment didn't decrease, since $\sum_{i} p_{i}^{\prime}-\sum_{i} p_{i}=d^{t} v \geq 0$. Now, since $x+d$ is not in the boundary of the polytope, we can give some more of good $g$ to some players without charging extra payments and increase their utility. Therefore $(x, p)$ is not Pareto-optimal.

For the $\Leftarrow$ direction, suppose $\left(x^{\prime}, p^{\prime}\right)$ is a Pareto improvement. Define $d=x^{\prime}-x$. First we claim that $d^{t} v>0$. By the definition of Pareto optimality, $v_{i} x_{i}-p_{i} \leq v_{i} x_{i}^{\prime}-p_{i}^{\prime}, \sum_{i} p_{i} \leq \sum_{i} p_{i}^{\prime}$ and at least one inequality is strict. Summing them all, we get that $\sum_{i} v_{i} x_{i}<\sum_{i} v_{i} x_{i}^{\prime}$, which implies that $d^{t} v>0$. Now, consider two cases:

If $x_{i}^{\prime} \leq x_{i}$ for all $i$ with $p_{i}=B_{i}$, then $d_{i} \leq 0$ for all such $i$. Simply pick some $i$ for which $d_{i}>0$ and decrease $d_{i}$ slightly. The result will be a dominated direction $d^{\prime}$ (since $x+d^{\prime} \leq x+d$ and $\left.x+d^{\prime} \neq x+d\right)$ with $d^{\prime t} v>0$ and $d_{i}^{\prime} \leq 0$ for all $i$ with $p_{i}=B_{i}$.

If $x_{i}^{\prime}>x_{i}$ for some $i$ with $p_{i}=B_{i}$. Then define $d^{\prime}$ such that $d_{i}^{\prime}=d_{i}$ if $p_{i}<B_{i}$ and $d_{i}^{\prime}=\min \left\{0, d_{i}\right\}$ if $p_{i}=B_{i}$. Now, consider $x^{\prime \prime}=x+d^{\prime}$ and $p^{\prime \prime}=p^{\prime}$. Clearly $d^{\prime}$ is a dominated direction (since

\footnotetext{
${ }^{3}$ This is without loss of genearality if one is allowed to randomize between outcomes, and can allocate lesser amount of the good $g$ to any player.
} 
$x+d^{\prime} \leq x+d$ and $\left.x+d^{\prime} \neq x+d\right)$ and $d_{i}^{\prime} \leq 0$ for $p_{i}=B_{i}$ by definition. Now, we will show that $d^{\prime t} v \geq 0$. Notice that $\sum_{i} p_{i}^{\prime \prime}=\sum_{i} p_{i}^{\prime} \geq \sum_{i} p_{i}$. Also, except for $i$ with $x_{i}^{\prime}>x_{i}$ and $p_{i}=B_{i}$ we have: $v_{i} x_{i}^{\prime \prime}-p_{i}^{\prime \prime}=v_{i} x_{i}^{\prime}-p_{i}^{\prime} \geq v_{i} x_{i}-p_{i}$. For theremaining $i$, one has: $v_{i} x_{i}^{\prime \prime}-p_{i}^{\prime \prime}=v_{i} x_{i}-p_{i}^{\prime} \geq v_{i} x_{i}-p_{i}$. Summing all those inequalities, we get $\sum_{i} v_{i} x_{i}^{\prime \prime} \geq \sum_{i} v_{i} x_{i}$, implying that $d^{\prime t} v \geq 0$.

Another simple observation is that if $(x, p)$ is a Pareto-optimal outcome in which no budget is fully exhausted, then $x=\operatorname{argmax}_{x \in X} v^{t} x$. For small valuations, any Pareto-optimal mechanism that satisfies individually rationality cannot exhaust budgets, so it must behave like VCG.

\subsection{Polymatroidal environments}

Our most interesting applications correspond to settings where the environment is a packing polytope $P=\left\{x \in \mathbb{R}_{+}^{n} ; A x \leq b\right\}$ for some $m \times n$ matrix with $A_{i j} \geq 0$ and $b \in \mathbb{R}_{+}^{m}$. We call such environments a polyhedral environment. Examples of polyhedral environments are ubiquitous in game theory (see [26, 27] for many examples).

A rich subclass of packing polytopes is the class of polymatroids, which are polytopes that can be written as $P=\left\{x \in \mathbb{R}_{+}^{n} ; \sum_{i \in S} x_{i} \leq f(S)\right\}$ where $f: 2^{[n]} \rightarrow \mathbb{R}_{+}$is a monotone submodular function, i.e., a function satisfying:

$$
\begin{gathered}
f(S \cup T)+f(S \cap T) \leq f(S)+f(T), \forall S, T \subseteq[n] \\
f(S) \leq f(T), \forall S \subseteq T \subseteq[n]
\end{gathered}
$$

Such polymatroidal environments generalize matroid environments. It is easy to see that all previously studied settings are instances of a these environments: Dobzinski et al's result [14] corresponds to the uniform matroid and Fiat et al's result [18] corresponds to the transversal matroid. Bikhchandani et al [8] give many examples of polymatroid environments including scheduling with due dates, network planning, pairwise kidney exchange, spatial markets, bandwidth markets and multi-class queueing systems [8]. In section 4 we discuss some of those applications in more depth and present a novel application of polymatroids to sponsored search auctions.

\section{Clinching Auction for Polymatroids}

In this section, we describe our main positive result, i.e., an auction with all the desirable properties for polymatroidal environments. This auction is based on the clinching auctions framework of Ausubel [5]. Before we study more complicated constraints, let's recall the clinching auction [5, 14] for the multi-unit setting, i.e., $P=\left\{x ; \sum_{i} x_{i} \leq s_{0}\right\}$. We begin by setting the supply $s=s_{0}$ and $B_{i}$ the budget available to each agent. We maintain a price clock $p$ that begins at zero and gradually ascends. For each price $p$, the agents are asked how much of the good they demand at

the current price. Their demand will be $d_{i}=\frac{B_{i}}{p}$ (how much they can afford with their remaining budget) if $p \leq v_{i}$, and zero otherwise (the case where the price exceeds their marginal value). Then agent $i$ is able to clinch an amount $\delta_{i}=\left[s-\sum_{j \neq i} d_{j}\right]^{+}$, which is the minimum amount we can give to player $i$ while we are still able to meet the aggregate demands of the other players. Clinching means that player $i$ gets $\delta_{i}$ amounts of the good, and $\delta_{i} p$ is subtracted from his budget. The price increases and we repeat the process until the supply is completely sold.

The heart of the mechanism is the clinching step and generalizing it for more complicated environments involves various challenges: how does one define the notion of supply and aggregate demand (it is not a single number anymore, since there are constraints restraining the possible allocation)? Finally, we need to make sure the clinching step doesn't violate feasibility. 
Clinching Framework. First, in Algorithm 1, we consider a slightly modified version of the clinching framework: we maintain a price vector $p \in \mathbb{R}_{+}^{n}$ and increase the prices one player at a time. The vector $\rho \in \mathbb{R}_{+}^{n}$ contains the promised allocations in each step and its final value is the final allocation of the mechanism. The payment of each agent is the total amount that was deducted from their budget during the execution 4 .

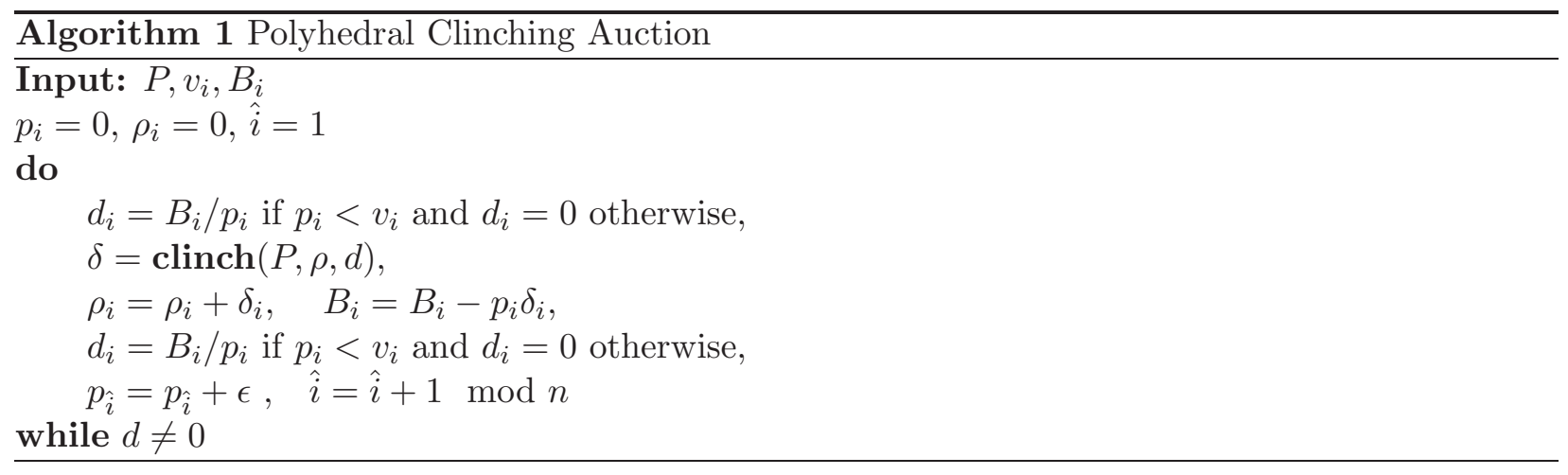

For each price, we calculate the demand $d_{i}$ of each player, which is the amount of the good they would like to get for price $p$. Then we invoke a procedure called clinch which decides the amount to grant to each player at that price. We update the promises, remaining budget and adjust demands5. Then we increase the price.

In order to define clinching, we need to define analogues of the remnant supply and to demands for the case where the the environment is a generic polytope. Instead of being a single number as in the multi-unit auctions case, the remnant supply and aggregate demands will be polytopes:

Definition 3.1 (aggregate demands) Given $P$, a vector of promised allocation $\rho \in P$, the remnant supply is described by the polytope $P_{\rho}=\{x \geq 0 ; \rho+x \in P\}$. If $d \in \mathbb{R}_{+}^{n}$ is the demand vector, the aggregate demand is defined by $P_{\rho, d}=\{x \geq 0 ; \rho+x \in P, x \leq d\}$.

In the multi-unit auctions case, the amount player $i$ clinched was the maximum amount we could give him while still being able to meet the demands of the other players. We generalize this notion to polyhedral environments (the concepts are depicted in Figure 10):

Definition 3.2 (polyhedral clinching) The demand set of players $[n] \backslash i$ if one allocates $x_{i}$ to player $i$ is represented by the polytope $P_{\rho, d}^{i}\left(x_{i}\right)=\left\{x_{-i} \in \mathbb{R}_{+}^{[n] \backslash i} ;\left(x_{i}, x_{-i}\right) \in P_{\rho, d}\right\}$. Since $P$ is a packing polytope, clearly $P_{\rho, d}^{i}\left(x_{i}\right) \supseteq P_{\rho, d}^{i}\left(x_{i}^{\prime}\right)$ if $x_{i} \leq x_{i}^{\prime}$. The amount player $i$ is able to clinch is the maximum amount we can give him without making any allocation for the other players infeasible. More formally, $\delta_{i}=\sup \left\{x_{i} \geq 0 ; P_{\rho, d}^{i}\left(x_{i}\right)=P_{\rho, d}^{i}(0)\right\}$.

We need to ensure that the clinching step is well-defined, i.e., that after clinching is performed, the vector of promised allocations is still feasible. This is done by the following lemma:

Lemma 3.3 For each step of the auction above, if $\rho \in P$, then $\rho+\delta \in P$.

Proof : Let $\chi^{i}$ be the $i$-th coordinate vector. Note that $\delta_{1} \chi^{1} \in P_{\rho, d}$ by definition of $\delta$. Now, notice that $\delta_{1} \chi^{1} \in P_{\rho, d}^{2}(0)=P_{\rho, d}^{2}\left(\delta_{2}\right)$, so: $\delta^{1} \chi_{1}+\delta_{2} \chi^{2} \in P_{\rho, d}$. By induction, we can show that

\footnotetext{
${ }^{4}$ Note that the details of the main procedure clinch is not described in this algorithm.

${ }^{5}$ Clearly updating demands is not necessary at this point, but we do in order to make the analysis cleaner.
} 

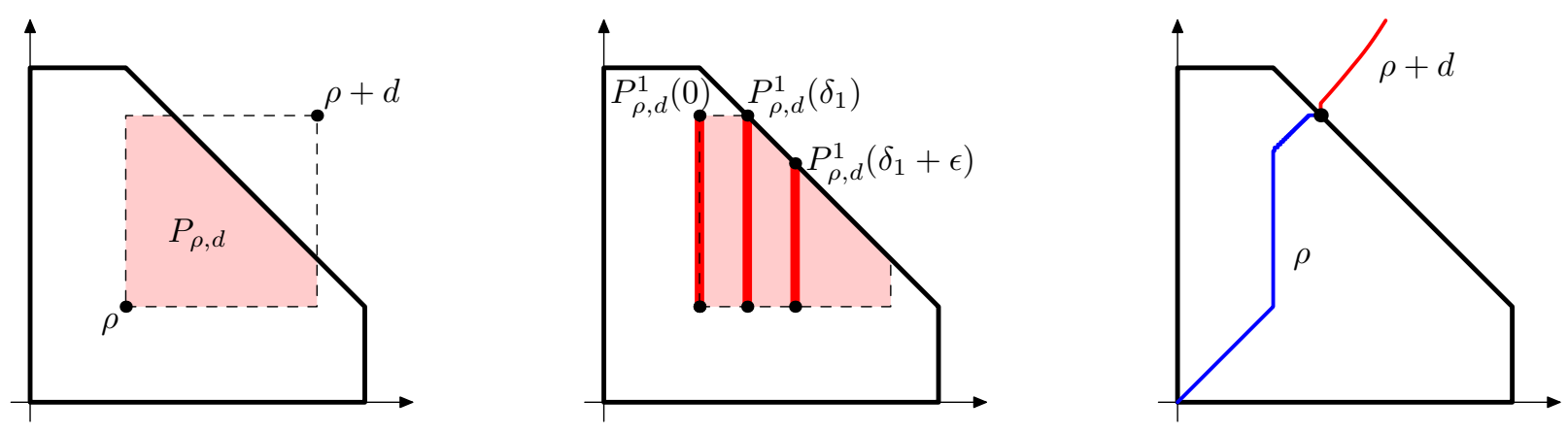

Figure 1: Illustration of polyhedral clinching: the first two figures depict the polytopes defined in Definitions 3.1 and 3.2. The third depicts the mechanism running on polytope $P$ : during the execution, the vector $\rho$ walks inside the polytope (blue line) and the vector $\rho+d$ walks outside it (red line), until they meet at the boundary. The point they meet corresponds to the final allocation.

$\sum_{i=1}^{j} \delta_{i} \chi^{i} \in P_{\rho, d}$. The induction is easy: $\sum_{i=1}^{j} \delta_{i} \chi^{i} \in P_{\rho, d}^{j+1}(0)=P_{\rho, d}^{j+1}\left(\delta_{j+1}\right)$, so $\sum_{i=1}^{j+1} \delta_{i} \chi^{i} \in P_{\rho, d}^{j+1}$.

This auction is clearly truthful, since each player $i$ reports only $v_{i}$, and she can stop her participation earlier (which she doesn't want, since she will potentially miss items she are interested in) or later (which will potentially give her items for a price higher than her valuation). It is also individually rational, since players only get items for prices below their valuation and respect budgets by the definition. Notice that those facts are true regardless of the trajectory of the price vector: any process that increases prices (in a potentially non-uniform way) has this property.

Lemma 3.4 The auction in Algorithm 1 along with the clinching step described in Definition 3.2 is truthful, individually-rational and budget-feasible.

Clinching for polymatroids. Notice that we haven't used anything from polymatroids yet, so Lemma 3.4 holds for any polytope $P$. However, two things are left to be shown: (i) that amount clinched can be computed efficiently and (ii) that the outcome is Pareto optimal. To show both of these properties, we use the fact that $P$ is a polymatroid.

Lemma 3.5 If the environment is a polymatroid $P$ defined by a submodular function $f$, then the amount player $i$ clinches in Algorithm 1 is given by:

$$
\delta_{i}=\left(\max _{x \in P_{\rho, d}} \mathbb{1}^{t} x\right)-\left(\max _{x \in P_{\rho, d}} \mathbb{1}_{-i}^{t} x_{-i}\right) .
$$

Moreover, this can be calculated efficiently using submodular minimization.

The main ingredients of the proof are the following two facts about polymatroids:

Fact 3.6 (Schrijver [30], sections 44.1 and 44.4) If $P$ is a polymatroid defined by the submodular function $f$, then $P_{\rho, d}$ is also a polymatroid defined by the following submodular function:

$$
\hat{f}(S)=\min _{T \subseteq S}\{f(T)-\rho(T)+d(S \backslash T)\} .
$$

Notice that $\hat{f}(\cdot)$ might not be monotone. However,

$$
\bar{f}(S)=\min _{S^{\prime} \supseteq S} \hat{f}\left(S^{\prime}\right)
$$


is a monotone submodular function that defines the same polymatroid.

Fact 3.7 Given two monotone submodular functions $f, \tilde{f}$, then the polymatroids $P, \tilde{P}$ defined by them are equal iff the functions are equal. The $(\Leftarrow)$ direction is trivial. For the other direction, notice that if $f(S)<\tilde{f}(S)$ say for $S=\{1, \ldots, i\}$, notice that the point $x$ such that $x_{j}=\tilde{f}(\{1 . . j\})-$ $\tilde{f}(\{1 . . j-1\})$ for $j \leq i$ and zero otherwise is such that $x \in \tilde{P} \backslash P$.

Proof of Lemma 3.5 : Using the fact 3.6, we know that $P_{\rho, d}^{i}\left(x_{i}\right)$ is also a polymatroid defined over $[n] \backslash i$ by the function $\tilde{f}(S)=\min \left\{\bar{f}(S), \bar{f}(S \cup i)-x_{i}\right\}$. Now, we use Fact 3.7 to see that $P_{\rho, d}^{i}\left(x_{i}\right)=P_{\rho, d}^{i}(0)$ iff $\bar{f}(S) \leq \bar{f}(S \cup i)-x_{i}, \forall S \subseteq[n] \backslash i$. So, $\delta_{i}=\min _{S \subseteq[n] \backslash i} \bar{f}(S \cup i)-\bar{f}(S)$. Since $\bar{f}$ is submodular, the smallest marginal can only be

$$
\bar{f}([n])-\bar{f}([n] \backslash i)=\max \{0, \hat{f}([n])-\hat{f}([n] \backslash i)\}
$$

which is exactly the expression in the statement of the lemma. Now, one can easily see that evaluating $\hat{f}$ is a submodular minimization problem.

Now we prove that the outcomes are Pareto-optimal in two steps. The first step is to characterize Pareto-optimal allocations for polymatroids. This characterization is stronger than that of Lemma 2.1. since it explores the structure of polymatroids. Afterwards, we show that the outcomes of the chinching auction defined in Algorithm 1 satisfy the two conditions in the characterization lemma.

In the following, for a vector $x \in \mathbb{R}^{n}$ and $S \subseteq[n]$ we denote $x(S)=\sum_{i \in S} x_{i}$.

Lemma 3.8 For a polymatroidal environment $P$ defined by a submodular function $f$, an allocation $(x, p)$ is Pareto optimal iff:

1. All items are sold, i.e., $x([n])=f([n])$, and

2. Given a player $i$ with $p_{i}<B_{i}$ and player $j$ with $v_{j}<v_{i}$, then there exists a set $S$ such that $x(S)=f(S), i \in S$ and $j \notin S$

The following elementary facts about submodular functions will be useful in the proof of the Lemma 3.8 .

Fact 3.9 Given a vector $x \in P$, if two sets $S, T$ are tight (i.e. $x(S)=f(S)$ and $x(T)=f(T)$ ), then $S \cap T$ and $S \cup T$ are also tight. The proof is quite elementary: $x(S \cup T)=x(S)+x(T)-$ $x(S \cap T) \geq f(S)+f(T)-f(S \cap T) \geq f(S \cup T)$. So, all the inequalities must be tight and therefore $x(S \cup T)=f(S \cup T)$ and $x(S \cap T)=f(S \cap T)$.

Fact 3.10 If $x([n])<f([n])$ then there is one component $x_{i}$ that we can increase by $\delta>0$ such that $x$ is still in $P$. It follows from the previous fact: if all players $i$ were contained in a tight set, one could take the union of those and $[n]$ would be tight. Then there is some element $i$ which is in no tight set.

Proof of Lemma 3.8 : The $(\Rightarrow)$ direction is easy. If $x([n])<f([n])$ then we can increase some $x_{i}$ (Fact 3.10) and still get point $P$ generating a Pareto improvement. Also, if there is $p_{i}<B_{i}$ and $v_{j}<v_{i}$ and no tight set separating them, then we can consider another outcome where we increase $x_{i}$ by some $\delta>0$, decrease $x_{j}$ by some $\delta<0$ and still get a feasible point improving $x^{t} v$. Now, this would not be Pareto optimal by Lemma 2.1.

For the $(\Leftarrow)$ direction, let $(x, p)$ be an outcome satisfying properties 1 and 2 and suppose $\left(x^{\prime}, p^{\prime}\right)$ is a Pareto-improvement. This means that $v_{i} x_{i}^{\prime}-p_{i}^{\prime} \geq v_{i} x_{i}-p_{i}$ and $\sum_{i} p_{i}^{\prime} \geq \sum_{i} p_{i}$. 
Let $\left\{i_{1}, \ldots, i_{k}\right\}=\{n\} \cup\left\{i ; p_{i}<B_{i}\right\}$, sorted in non-increasing order of $v_{i}$. Using property 2 (notice it holds for player $n$ trivially) together with fact 3.9, we define the following family of tight sets $S_{1} \subseteq S_{2} \subseteq \ldots \subseteq S_{k}=[n]$, tight in the sense that $x\left(S_{i}\right)=f\left(S_{i}\right)$. For all $v_{t}<v_{i_{1}}$ there is a tight set $S_{1 t}$ that has $i_{1}$ but not $t$. Let $S_{1}$ be the intersection of such sets. Now, given $S_{1} \subseteq \ldots \subseteq S_{j-1}$, we define $S_{j}$ in the following way: If $i_{j} \in S_{j-1}$, take $S_{j}=S_{j-1}$ (notice can only happen if $v_{i_{j}}=v_{i_{j-1}}$ ). If not, for each $v_{t}<v_{i_{j}}$ there is a tight set $S_{j t}$ that has $i_{j}$ but not $t$. Now, define $S_{j}$ as the union of $S_{j-1}$ and the intersection of the $S_{j t}$ sets.

By eliminating duplicates and its corresponding elements from $\left\{i_{1} \ldots i_{k}\right\}$, we get a family $S_{1} \subset$ $\ldots \subset S_{k}$. Define $T_{j}=S_{j} \backslash S_{j-1}$ and it is clear the family obtained has the following properties:

- all $t \in S_{j}$ have $v_{t} \geq v_{i_{j}}$

- $i_{j} \in T_{j}$

- for all $i \in T_{j}$ either $v_{i}=v_{i_{j}}$ or $p_{i}=B_{i}$.

Let $T_{j}^{\prime}=\left\{i \in T_{j} ; v_{i} \neq v_{i_{j}}\right\}$ and $T_{j}^{\prime \prime}=\left\{i \in T_{j} ; v_{i}=v_{i_{j}}\right\}$. Since the players in $T_{j}^{\prime}$ have exhausted their budget, $p_{i} \geq p_{i}^{\prime}$. Using that and Pareto-optimality, we get:

$$
\begin{aligned}
& \sum_{i \in T_{j}^{\prime}} p_{i}-p_{i}^{\prime} \geq \\
& \quad \geq \sum_{i \in T_{j}^{\prime}, x_{i} \geq x_{i}^{\prime}} p_{i}-p_{i}^{\prime} \geq \sum_{i \in T_{j}^{\prime}, x_{i} \geq x_{i}^{\prime}} v_{i}\left(x_{i}-x_{i}^{\prime}\right) \geq \\
& \geq \sum_{i \in T_{j}^{\prime}, x_{i} \geq x_{i}^{\prime}} v_{i_{j}}\left(x_{i}-x_{i}^{\prime}\right) \stackrel{* *}{\geq} \sum_{i \in T_{j}^{\prime}} v_{i_{j}}\left(x_{i}-x_{i}^{\prime}\right)
\end{aligned}
$$

Now, we can add the inequality $p_{i}-p_{i}^{\prime} \geq v_{i_{j}}\left(x_{i}-x_{i}^{\prime}\right)$ for $i \in T_{j}^{\prime \prime}$ and obtain:

$$
\sum_{i \in T_{j}} p_{i}-p_{i}^{\prime} \geq \sum_{i \in T_{j}} v_{i_{j}}\left(x_{i}-x_{i}^{\prime}\right)
$$

Summing those for all $j$ and get:

$$
\sum_{i} p_{i}-p_{i}^{\prime} \geq \sum_{j} \sum_{i \in T_{j}} v_{i_{j}}\left(x_{i}-x_{i}^{\prime}\right)=\sum_{j}\left(v_{i_{j}}-v_{i_{j+1}}\right) \sum_{i \in S_{j}}\left(x_{i}-x_{i}^{\prime}\right) \geq 0
$$

since $x\left(S_{j}\right)=f\left(S_{j}\right) \geq x^{\prime}\left(S_{j}\right)$. Therefore $\sum_{i} p_{i} \geq \sum_{i} p_{i}^{\prime}$ and therefore equal. This means in particular all of the inequalities in (11) and (2) must be tight. Therefore for all $i \in T_{j}^{\prime}$ we need to have $x_{i}=x_{i}^{\prime}$, since if $x_{i}>x_{i}^{\prime}$ then inequality $*$ in (11) would be strict. If $x_{i}<x_{i}^{\prime}$, then inequality ** would be strict. We use this fact to show that $\sum_{i \in S_{j}} v_{i}\left(x_{i}-x_{i}^{\prime}\right) \geq 0$ by induction on $j$. If we show that, we can take $j=k$ and then we are done, since this will imply that $\sum_{i} v_{i} x_{i} \geq \sum_{i} v_{i} x_{i}^{\prime}$ and therefore $\left(x^{\prime}, p^{\prime}\right)$ cannot be a Pareto-improvement.

For $j=1$, this is trivial, since we can write:

$$
\sum_{i \in S_{1}, v_{i} \neq v_{i_{1}}} v_{i}\left(x_{i}-x_{i}^{\prime}\right) \geq \sum_{i \in S_{1}, v_{i} \neq v_{i_{1}}} v_{i_{1}}\left(x_{i}-x_{i}^{\prime}\right)
$$


since both terms are zero, and then sum $v_{i}\left(x_{i}-x_{i}^{\prime}\right)$ for the rest of the elements in $S_{1}$ and use the fact that $S_{1}$ is tight. For other $j$, we use that:

$$
\begin{aligned}
& \sum_{i \in S_{j}} v_{i}\left(x_{i}-x_{i}^{\prime}\right) \geq \\
& \quad \geq v_{i_{j-1}} \sum_{i \in S_{j-1}}\left(x_{i}-x_{i}^{\prime}\right)+\sum_{i \in T_{j}^{\prime}} v_{i}\left(x_{i}-x_{i}^{\prime}\right)+\sum_{i \in T_{j}^{\prime \prime}} v_{i_{j}}\left(x_{i}-x_{i}^{\prime}\right) \geq \\
& \quad \geq v_{i_{j}} \sum_{i \in S_{j}}\left(x_{i}-x_{i}^{\prime}\right)=v_{i_{j}}\left(x\left(S_{j}\right)-x^{\prime}\left(S_{j}\right)\right) \geq 0,
\end{aligned}
$$

by the fact that $S_{j}$ is tight.

Now, we argue that, for sufficiently small $\epsilon$, the outcome satisfied the two properties in Lemma 3.8 and hence is Pareto-optimal. We prove this fact using the following sequence of lemmas:

Lemma 3.11 After the clinching step is executed, and before updating prices, $\hat{f}([n]) \leq \hat{f}([n] \backslash$ $j), \forall j \in[n]$.

Proof : In the clinching step, given an initial $\hat{f}_{0}$, we define $\delta_{i}=\max \left\{0, \hat{f}_{0}([n])-\hat{f}_{0}([n] \backslash i)\right\}$. After we update $\rho, B, d, \hat{f}$ is updated to $\hat{f}_{1}(S)=\hat{f}_{0}(S)-\delta(S)$. Now, it is easy to check that:

$$
\hat{f}_{1}([n])=\hat{f}_{0}([n])-\sum_{i} \delta_{i}=\hat{f}_{0}([n])-\delta_{j}-\sum_{i \neq j} \delta_{i} \leq \hat{f}_{0}([n] \backslash j)-\sum_{i \neq j} \delta_{i}=\hat{f}_{1}([n] \backslash j)
$$

Lemma 3.12 The outcome $(x, p)$ of the clinching auction is such that $x([n])=f([n])$.

Proof : We show the following invariant: if we define $\hat{f}$ as in Fact 3.6, updating it each round as $\rho, d$ changes, we claim that the value of $\mathbb{1}^{t} \rho+\hat{f}([n])$ remains constant.

To do so, we consider the events that can cause it to drop:

1. clinching: just after clinching occurs (i.e. $\rho_{i}$ increases by $\delta_{i}$, budgets decrease by $p \delta_{i}$, demands are adjusted, but before the price increases), the amount $\mathbb{1}^{t} \rho+\hat{f}([n])$ remains the same since $\rho^{t} \mathbb{1}$ increases by $\delta^{t} \mathbb{1}$ and for all $S, \hat{f}(S)$ decreases by $\delta(S)$, because to each $i, \rho$ increases by $\delta_{i}$ and $d_{i}$ decreases by $\delta_{i}$.

2. price $p_{i}$ increases and $d_{i}$ decreases by $\theta, 0 \leq \theta \leq d_{i}$. If $\mathbb{1}^{t} \rho+\hat{f}([n])$ decreased then there was some $T, i \notin T$ such that:

$$
\hat{f}([n] \backslash i)+d_{i}-\theta \leq f(T)-\rho(T)+d([n] \backslash T)-\theta<\hat{f}([n])
$$

Using Lemma 3.11, we know that $\hat{f}([n]) \leq \hat{f}([n] \backslash i)$, so $d_{i}<\theta$ which is not true.

The proofs of the previous two lemmas intuitively establishes the maximality of the clinching procedure. Lemma 3.11 can be interpreted as saying that if we apply the clinching procedure twice, without updating prices, then the second time will have no effect. The proof of Lemma 3.12 identifies an invariant that is maintained during the execution of the mechanism. 
Lemma 3.13 If $\epsilon<\min _{v_{i} \neq v_{j}}\left|v_{i}-v_{j}\right|$, then property 2 of Lemma 3.8 is satisfied.

Proof : Suppose not and for the final outcome there are $v_{j}<v_{i}, p_{i}<B_{i}$ and all sets $S$ such that $i \in S, j \notin S$ are not tight. First, clearly $x_{j} \neq 0$, otherwise $[n] \backslash j$ would be tight by Lemma 3.12 . Then consider $\tilde{x}$ where $\tilde{x}_{i}=x_{i}+\theta, \tilde{x}_{j}=x_{j}-\theta$ and $\tilde{x}_{k}=x_{k}$ for all $k \neq i, j$. It is feasible for some small $\theta$.

Now, consider the promised allocation $\rho$ and demands $d$ just before the last time player $j$ clinched an amount $\delta_{j}>0$. If necessary decrease $\theta$ so that it becomes smaller than this last amount clinched, i.e., $\theta<\delta_{j}$. At this point $\rho \leq x \leq \rho+d$. By the definition of clinching: $P_{\rho, d}^{j}\left(\delta_{j}\right)=P_{\rho, d}^{j}(\theta)=P_{\rho, d}^{j}(0)$.

At this point, $\rho \leq x$ and $\rho_{j}+\theta<\rho_{j}+\delta_{j}=x_{j}$. Therefore $\tilde{x} \geq \rho$. Also, we have that $x-\rho \leq d$ and $x_{i}-\rho_{i}<d_{i}$, since agent $i$ hasn't dropped his demand to zero yet and his demand never increases and won't be met while $v_{i}<p_{i}$. Here we are strongly using that $\epsilon<\min _{v_{i} \neq v_{j}}\left|v_{i}-v_{j}\right|$ to ensure that for the last time player $j$ clinches, player $i$ demand is not zero yet. This implies that $\tilde{x}-\rho \in P_{\rho, d}$ so $(\tilde{x}-\rho)_{-j} \in P_{\rho, d}^{j}(0)$. Now, the fact that $P_{\rho, d}^{j}(0)=P_{\rho, d}^{j}\left(\delta_{i}\right)$ implies that $\hat{x}=\left(x_{j}, \tilde{x}_{-j}\right) \in P$. But $\hat{x}([n])=x([n])+\theta=f([n])+\theta>f([n])$, which is an absurd.

We can summarize the results as:

Theorem 3.14 For a polymatroidal environment, the auction in Algorithm 1 along with the clinching step described in Definition 3.2 has all the desirable properties.

Extensions and limitations of the clinching framework: The clinching framework described in Algorithm 1 and Definition 3.2 is quite flexible: one can change the way clinching is done or the way prices ascend and obtain an auction that is still truthful, individually rational, and respects budgets. Pareto-optimality, however, is a delicate property to achieve. A natural question is for which environments Pareto-optimality is still achievable? In appendix A, we show we can extend this framework a little further (to scaled polymatroids), but not further than that.

Faster clinching: We showed in this section that for a generic polymatroid, we can calculate the clinched amount using submodular minimization as a sub-routine. For each individual environment, however, one can usually find much faster clinching subroutines. We illustrate this in appendix B for the single-keyword AdWords polytope.

\section{The AdWords Polytope and other applications}

We begin by discussing some interesting applications of the auction presented in section 3 , Then we introduce a novel application of polymatroidal constraints to sponsored search auctions that generalizes the classical models of Edelman et al [16] and Varian [31].

- Multi-unit auctions [14]: corresponds to the polymatroids associated with constant submodular functions, i.e., $f(S)=Q, \forall S$.

- Combinatorial auctions with matching constraints [18]: there is a bipartite graph $([m],[n], E)$ between items $[m]$ and bidders $[n]$ and each buyer $i$ has additive value 1 for each item $j$ such that $(i, j) \in E$ and value 0 for each item not connected to him. We can represent this setting by a polymatroid where $f(S)$ is the number of items connected to some player in $S$. This is called the transversal matroid. 
- Video on demand [8]: Consider company that provides video on demand that is located on a node $s$ of a direct network with capacities on the edges $G=(V, E, c)$. Each buyer corresponds to a node in the network. An allocation $x$ is feasible if it is possible to transmit at rate $x_{i}$ for each player $i$ simultaneously. This is possible if for each subset $S \subseteq[n]$ of players, $\sum_{i \in S} x_{i}$ is smaller then the min-cut from $s$ to $S$. Using the submodularity of the cut-function, it is easy to see that the environment is a polymatroid.

- Spanning tree auctions: Consider the abstract setting where the agents are edges of a graph $G$ and the auctioneer is allowed to allocate goods to a set only if it has no cycles. This corresponds to the graphical matroid of graph $G$. A more practical setting is when a telecommunication company owns a network that contains cycles and decides to auction their redundant edges. This setting corresponds to the dual-graphical matroid of $G$.

\subsection{AdWords Polytope}

Consider $n$ advertisers and $m$ keywords. Each advertiser $i$ is interested in a subset of the keywords $\Gamma(i) \subseteq[m]$. For a keyword $k$, we denote by $\Gamma(k)$, the set of advertisers interested in this keyword. With each keyword $k$, we associate $|\Gamma(k)|$ positions. Position $j$ for keyword $k$ has click-through-rate $\alpha_{j}^{k}$ (possibly zero) such that $\alpha_{1}^{k} \geq \alpha_{2}^{k} \geq \ldots \geq \alpha_{|\Gamma(k)|}^{k}$ for each $k$.

Assuming that each keyword gets a large amount of queries, we see $\alpha_{j}^{k}$ as the sum of number of clicks that the $j$-th position of keyword $k$ gets across all queries that it matches. For now, let's assume that the number of clicks a player gets in slot $j$ of keyword $k$ depends only on $j, k$ and not on the identity of the player. One is able to relax this assumption, as we see later.

Let $\mathcal{A}_{k}=\left\{\pi_{k}: \Gamma(k) \hookrightarrow[|\Gamma(k)|]\right\}$ be the set of all allocations (one-to-one maps) from players to slots for keyword $k$. Also, let $\Delta\left(\mathcal{A}_{k}\right)$ be the distributions of such allocations. Given that, we can define the AdWords polytope in the following way: an allocation of clicks $x$ is feasible if there is a distribution over allocations of players to slots for each keyword such that player $i$ gets $x_{i}$ clicks in expectation. More formally:

Definition 4.1 (AdWords Polytope) The AdWords polytope is the set of feasible allocations 6 of clicks $x=\left(x_{1}, \ldots, x_{n}\right)$ such that there are distributions $\mathcal{D}_{k} \in \Delta\left(\mathcal{A}_{k}\right)$ for each keyword, and

$$
x_{i} \leq \sum_{k \in \Gamma(i)} \mathbb{E}_{\pi_{k} \sim \mathcal{D}_{k}}\left[\alpha_{\pi_{k}(i)}^{k}\right]
$$

Our main result in this Section is that:

Theorem 4.2 The AdWords polytope is a polymatroid.

In order to prove the theorem, we first consider the setting with a single keyword and all advertisers interested in it. Let the click-through-rates be $\alpha_{1} \geq \ldots \geq \alpha_{n}$. Feldman et al [17] relate the problem of deciding if a vector $x$ is feasible to a classical problem in machine scheduling, i.e., scheduling in related machines with preemptions $\left(Q|p m t n| C_{\max }[19]\right)$. What follows is a restatement of their characterization in a format that makes it clear it is a polymatroidal environment.

Lemma 4.3 (Feldman et al [17]) An allocation vector $x$ is feasible iff for each $S, x(S) \leq \sum_{j=1}^{|S|} \alpha_{j}$, where $x(S)=\sum_{i \in S} x_{i}$ for each set $S \subseteq[n]$.

\footnotetext{
${ }^{6}$ Since the number of clicks is typically very large we treat them as divisible goods and consider also fractional allocations.
} 
Notice that $f_{k}(S)=\sum_{j=1}^{|S|} \alpha_{j}^{k}$ is a submodular function, so the set of feasible allocations for the single-keyword setting is a polymatroid.

For the multiple-keyword setting, we say that an allocation vector $x$ is feasible if we can write $x_{i}=\sum_{k \in \Gamma(i)} x_{i}^{k}$ in such a way that the vector $\left(x_{i}^{k}\right)_{i \in \Gamma(k)}$ is feasible for keyword $k$, i.e., $x^{k}(S) \leq f_{k}(S)$ for every $S \subseteq \Gamma(k)$.

The fact that this allocation set is a polymatroid is a direct consequence of the following theorem, which is a polymatroidal version of Rado's Theorem due to McDiarmid [24].

Theorem 4.4 (McDiarmid [24]) Given a bipartite graph $([n] \cup[m], E)$, its neighborhood map $\Gamma(\cdot), m$ submodular functions $f_{1}, \ldots, f_{m}$ and their respective polymatroids $P_{1}, \ldots, P_{m}$, then the set:

$$
P^{*}=\left\{x \in \mathbb{R}_{+}^{n} ; x_{i}=\sum_{k \in \Gamma(i)} x_{i}^{k} \text { and } x^{k} \in P_{k}\right\}
$$

is a polymatroid defined by the function

$$
f^{*}(S)=\sum_{k} f_{k}(S \cap \Gamma(k))
$$

Quality factors: So far, we assumed that the click-through-rate of player $i$ allocated to slot $j$ of keyword $k$ depends solely on $k$ and $j$. More generally, we would like to consider the click-throughrate of a slot depending also on the player allocated in that slot. Let $\alpha_{j, i}^{k}$ be the click-through-rate of position $j$ of keyword $j$ when player $i$ is placed there. Traditionally, we consider the click-throughrates in a product form, i.e., $\alpha_{j, i}^{k}=\alpha_{j}^{k} \cdot \gamma_{i}^{k}$ where $\gamma_{i}^{k}$ is called quality factor. Assuming quality factors are public information, one can, in a similar way, define a polytope of feasible allocations. In general it will not be a polymatroid.

If the quality factors are uniform among all queries, i.e., $\gamma_{i}^{k}=\gamma_{i}$, the the set of feasible allocations is given by $P_{\gamma}^{*}=\left\{x ;\left(\frac{x_{i}}{\gamma_{i}}\right)_{i} \in P^{*}\right\}$ where $P^{*}$ is the AdWords polytope defined as a function of $\alpha_{j}^{k}$. It is a scaled polymatroid, for which a variant of the auction in section 3 satisfies all desirable properties (see appendix A]).

\section{Limitations of auctions for budget-constrained agents}

Previously, we argued why simple modifications to the clinching auction would not work for polyhedral environments beyond (scaled) polymatroids. Here, we explore the possibility of designing an auction of a different format achieving those properties and show that this is not possible even for two players. We do so through a general characterization of Pareto-optimal auctions with desirable properties. Before stating the characterization, we study the case with one budget-constrained player and prove some lemmas that are useful in proving the general characterization result later.

\subsection{One budget-constrained player}

For ease of exposition, we first focus on 2 players and assume that the feasible set of allocations $P$ has a smooth and strictly-concave boundary, in the sense that for each $v \in \mathbb{R}_{+}^{2}$ there is a single point $x^{*}(v) \in P$ maximizing $v^{t} x$ such that $x^{*}(v)$ is a $\mathcal{C}^{\infty}$-function. In fact, one can approximate any polytope by such a set using the technique of Dolev et al [15. Using compactness arguments, it is 
possible to get an auction for the original environment by taking the limit of the auctions obtained for its $\mathcal{C}^{\infty}$-approximations.

Assume that player 1 is not budget constrained and player 2 has budget $B_{2}$ and let $\left(x^{*}, p^{*}\right)$ be the VCG mechanism for this setting. Now, we can define the function:

$$
\xi\left(v_{1}\right)=\min \left\{v_{2} ; p_{2}^{*}\left(v_{1}, v_{2}\right) \geq B_{2}\right\}
$$

Theorem 5.1 The allocation rule

$$
x\left(v_{1}, v_{2}\right)=x^{*}\left(v_{1}, \min \left\{v_{2}, \xi\left(v_{1}\right)\right\}\right)
$$

is monotone. Moreover, when coupled with the appropriate payment rule, it generates a Paretooptimal and budget feasible mechanism

Proof : The main part of the proof is to show that the allocation is monotone. If we show that, it is clearly budget feasible for player 2, since we use the VCG-payment rule until the point the budget of player 2 gets exhausted and from that point on, the allocation is constant. When the budgets of the players are not exhausted, the allocation is efficient (since it mimics VCG) and therefore is Pareto-optimal. The allocation when the budget of player 2 is exhausted is equivalent to the VCG allocation of a pair $\left(v_{1}, v_{2}^{\prime}\right)$ with $v_{2}^{\prime} \leq v_{2}$, so player 1 is getting $x_{1}^{*}\left(v_{1}, v_{2}^{\prime}\right) \geq x_{1}^{*}\left(v_{1}, v_{2}\right)$ by monotonicity of VCG. This implies Pareto-optimality as a consequence of Lemma 2.1.

Monotonicity: The allocation rule is clearly monotone for player 2. We need to show it is monotone for player 1 , i.e. that the function $t \mapsto x_{1}\left(v_{1}+t, v_{2}\right)$ is monotone non-decreasing. It is clearly so for intervals where $\xi\left(v_{1}+t\right) \geq v_{2}$, so let's assume that for $t \in(-\epsilon,+\epsilon)$ we have $\xi\left(v_{1}+t\right)<v_{2}$. Our goal is to show that: $\frac{d}{d t} x_{1}^{*}\left(v_{1}+t, \xi\left(v_{1}+t\right)\right) \geq 0$. Since the VCG-allocation lies in the boundary of $P$, this is the same as showing that $\frac{d}{d t} x_{2}^{*}\left(v_{1}+t, \xi\left(v_{1}+t\right)\right) \leq 0$. The crucial observation is that the VCG-payment for player 2 on the curve $\left(v_{1}+t, \xi\left(v_{1}+t\right)\right)$ is constant, i.e.:

$$
B_{2} \equiv p_{2}^{*}\left(v_{1}+t, \xi\left(v_{1}+t\right)\right)=\xi\left(v_{1}+t\right) x_{2}^{*}\left(v_{1}+t, \xi\left(v_{1}+t\right)\right)-\int_{0}^{\xi\left(v_{1}+t\right)} x_{2}^{*}\left(v_{1}+t, u\right) d u
$$

Now, we can simply derivate it with respect to $t$. We use the notation $\partial_{i} f(\cdot)$ for the derivative of $f$ with respect to the $i$-th variable. We also define $x_{2}^{*}(t)=x_{2}^{*}\left(v_{1}+t, \xi\left(v_{1}+t\right)\right)$. Now,

$$
\begin{gathered}
0=\xi^{\prime}\left(v_{1}+t\right) x_{2}^{*}(t)+\xi\left(v_{1}+t\right) \frac{d}{d t} x_{2}^{*}(t)-\xi^{\prime}\left(v_{1}+t\right) x_{2}^{*}(t)-\int_{0}^{\xi\left(v_{1}+t\right)} \partial_{1} x_{2}^{*}\left(v_{1}+t, u\right) d u \\
\xi\left(v_{1}+t\right) \frac{d}{d t} x_{2}^{*}(t)=\int_{0}^{\xi\left(v_{1}+t\right)} \partial_{1} x_{2}^{*}\left(v_{1}+t, u\right) d u \leq 0
\end{gathered}
$$

since $x_{2}^{*}\left(v_{1}, v_{2}\right)$ decreases with $v_{1}$ by the definition of the VCG allocation.

A variant of the proof can be used to show the following result for 2 budget constrained players. This is useful for our general characterization.

Corollary 5.2 If the functions $\xi_{1}\left(v_{2}\right), \xi_{2}\left(v_{1}\right)$ are such that the regions $\left\{v ; v_{2} \geq \xi_{2}\left(v_{1}\right)\right\}$ and $\left\{v ; v_{1} \geq\right.$ $\left.\xi_{1}\left(v_{2}\right)\right\}$ are disjoint, then one can define

$$
\begin{gathered}
x\left(v_{1}, v_{2}\right)=x^{*}\left(\min \left\{v_{1}, \xi_{1}\left(v_{2}\right)\right\}, \min \left\{v_{2}, \xi_{2}\left(v_{1}\right)\right\}\right) \\
p_{i}(v)=v_{i} x_{i}(v)-\int_{0}^{v_{i}} x_{i}\left(u, v_{-i}\right) d u .
\end{gathered}
$$

If $p_{2}\left(v_{1}, \xi_{2}\left(v_{1}\right)\right) \equiv B_{2}$ and $p_{1}\left(\xi_{1}\left(v_{2}\right), v_{2}\right) \equiv B_{1}$, then $(x, p)$ is a mechanism with the desirable properties. 
The above corollary has a strong fixed-point flavour and it is tempting to believe one could get the existence of such a mechanism from this theorem. This is however not true, as shown in the next section. However, this result remains useful as a tool for searching for such mechanisms whenever they exist. For example, one can extend the above theorem to prove the existence of the mechanisms for polyhedral environments when $B_{1}$ is much larger then $B_{2}$.

\subsection{Characterization and impossibility}

Now we discuss our main negative result: which states an impossibility of extending the auction for polymatroids to general polyhedral environments.

Theorem 5.3 (Impossibility) There is no general auction for every polyhedral environment and every pair of budgets that satisfies the desirable properties.

We prove it in two steps: first we prove a sequence of lemmas characterizing 2-player auctions for polyhedral environments satisfying all the desirable properties. Then we fix a specific polyhedral environment and argue that no mechanism can possibly satisfy this characterization.

First, we begin by understanding the format of an auction with the desirable properties where the environment is a packing polytope $P \subseteq \mathbb{R}_{+}^{2}$. We start by defining a family of VCG auctions. that

VCG-family: We say that a mechanism is in the $V C G$-family if its allocation $x(v) \in P$ is such

$$
x(v) \in X^{*}(v):=\operatorname{argmax}_{x \in P} v^{t} x .
$$

Notice that there might be more than one such mechanism: if $v$ is normal to an edge of the polytope, then the entire edge is in the argmax. Nevertheless, $x\left(v_{1}+, v_{2}\right)=\lim _{v_{1}^{\prime} \downarrow v_{1}} x\left(v_{1}^{\prime}, v_{2}\right)$ and $x\left(v_{1}, v_{2}+\right)=\lim _{v_{2}^{\prime} \downarrow v_{2}} x\left(v_{1}, v_{2}^{\prime}\right)$ are common for the entire family, as we see in the following lemma. A consequence of this fact is that the payment function might not be unique, but $p\left(v_{1}+, v_{2}\right)$ and $p\left(v_{1}, v_{2}+\right)$ are unique.

Lemma 5.4 Given a convex set $P \subseteq \mathbb{R}_{+}^{2}$ and two allocation rules $x(v), \tilde{x}(v) \in X^{*}(v):=\operatorname{argmax}_{x \in P} v^{t} x$, then $x\left(v_{1}+, v_{2}\right)=\tilde{x}\left(v_{1}+, v_{2}\right)$, where $x\left(v_{1}+, v_{2}\right)=\lim _{v_{1}^{\prime} \downarrow v_{1}} x\left(v_{1}^{\prime}, v_{2}\right)$.

The proof is elementary and can be found in Appendix C. To illustrate this fact, consider the simple case of $P=\left\{x \in \mathbb{R}_{+}^{2} ; x_{1}+x_{2} \leq 1\right\}$. Then the VCG mechanism is well-defined for $x_{1} \neq x_{2}$, which is, simply to allocate to the player with the highest value the entire amount. But notice that completing this mechanism with any allocation in the points $(v, v)$ generates a truthful mechanism. The payments of different mechanisms of the VCG family differ on $(v, v)$, for example $p_{i}(v, v)=v x_{i}(v)$, but notice that the payments everywhere else are well-defined.

Pareto-optimal mechanisms: Now we turn our attention back to Pareto-optimal mechanisms for two budget-constrained players. Let $(x, p)$ be such mechanism. As a direct consequence of the characterization of Pareto optimal outcomes (Lemma 2.1), we know the following:

- if $p_{i}(v)<B_{i}$ then $x_{i}(v) \geq \min \left\{x_{i} ; x \in X^{*}(v)\right\}$

- if $p_{1}(v)<B_{1}, p_{2}(v)<B_{2}$ then $x(v) \in X^{*}(v)$

And a simple consequence of truthfulness:

- if $p_{i}(v)=B_{i}$ then for all $v_{i}^{\prime} \geq v_{i}, x\left(v_{i}^{\prime}, v_{-i}\right)=x\left(v_{i}, v_{-i}\right)$. 


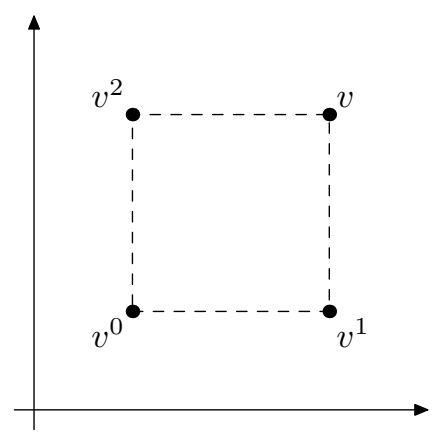

(i)

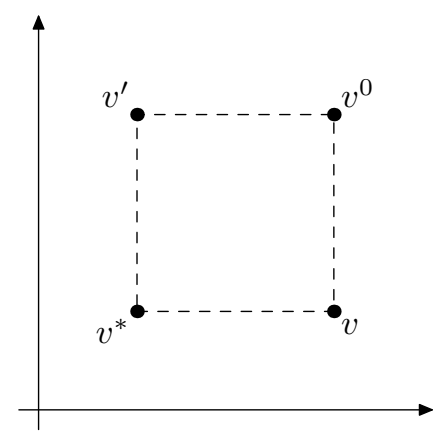

(ii)

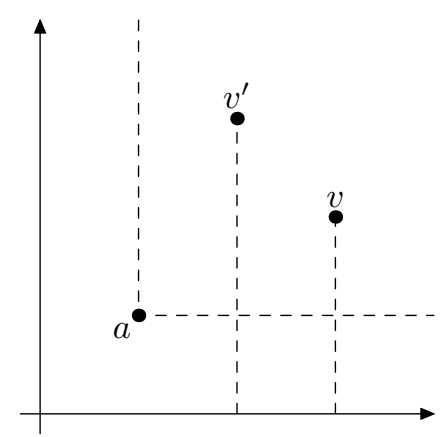

second-part

Figure 2: Illustration of the proof of Lemma 5.5

Now, we are ready to start proving the characterization theorem. We will characterize the mechanism in terms of the regions in the space of valuations where the budgets get exhausted. For formally, we are interested in understanding the sets:

$$
E_{i}=\left\{v \in \mathbb{R}_{+}^{2} ; p_{i}(v)=B_{i}\right\}
$$

Lemma 5.5 If mechanism $(x, p)$ has the desirable properties, then either $E_{1} \cap E_{2}=\emptyset$ or there exists some $a \in \mathbb{R}_{+}^{2}$ such that $\{v ; v>a\} \subseteq E_{1} \cap E_{2} \subseteq\{v ; v \geq a\}$.

Proof : Assume that $E_{1} \cap E_{2}$ is not empty. Then we will prove the lemma in two parts. For the first part we will prove two statements: (i) if that if $v^{0} \in E_{1} \cap E_{2}$ and $v \geq v^{0}$ then $x(v)=x\left(v^{0}\right)$ and (ii) if $v, v^{\prime} \in E_{1} \cap E_{2}$ and $v_{i}^{*}=\min \left\{v_{i}, v_{i}^{\prime}\right\}$ then $x\left(v^{*}\right)=x(v)=x\left(v^{\prime}\right)$. Then for the second part, we show that this whole region that has constant allocation has budget exhausted for the two players. See figure 2 for an illustration of the proof.

For (i), let $v^{1}=\left(v_{1}, v_{2}^{0}\right)$ and $v^{2}=\left(v_{1}^{0}, v_{2}\right)$ and notice that $x\left(v^{0}\right)=x\left(v^{1}\right)=x\left(v^{2}\right)$, since budgets are exhausted so the allocation can't increase. By monotonicity, $x_{2}(v) \geq x_{2}\left(v^{1}\right)=x_{2}\left(v^{0}\right)$ and $x_{1}(v) \geq x_{1}\left(v^{2}\right)=x_{1}\left(v^{0}\right)$. Since all allocations lie in the boundary of the polytope, we must have $x(v)=x\left(v^{0}\right)$.

The proof of (ii) is very similar, define $v_{i}^{0}=\max \left\{v_{i}, v_{i}^{\prime}\right\}$. Then by (i), $x(v)=x\left(v^{0}\right)=x\left(v^{\prime}\right)$ now, by the exact same argument as above we show that $x\left(v^{*}\right)=x\left(v^{0}\right)$.

Now, if $a_{i}=\inf \left\{v_{i} ; v \in E_{1} \cap E_{2}\right\}$, let us show that for $v>a, v \in E_{1} \cap E_{2}$. Let us show that $v \in E_{2}$ and then $E_{1}$ is analogous. By definition, there is some $v^{\prime} \in E_{1} \cap E_{2}$ with $v_{1}^{\prime}<v_{1}$. Then $\left(v_{1}^{\prime}, v_{2}\right) \in E_{2}$ since the allocation is constant, for $v>a$, the budget of 2 is exhausted in $\left(v_{1}^{\prime}, v_{2}\right)$ iff it is exhausted in $\left(v_{1}^{\prime}, v_{2}^{\prime}\right)$. Now, note that by monotonicity $x_{1}\left(v_{1}^{\prime}, u\right) \leq x_{1}\left(v_{1}, u\right)$ and since allocation is in the boundary of the polytope $x_{2}\left(v_{1}^{\prime}, u\right) \geq x_{2}\left(v_{1}, u\right)$. By the payment formula, $p_{2}(v)=v_{2} x_{2}(v)-\int_{0}^{v_{2}} x_{2}\left(v_{1}, u\right) d u$ and by the fact that $x_{2}(v)=x_{2}\left(v_{1}^{\prime}, v_{2}\right)$, we have that $B_{2} \geq p_{2}(v) \geq p_{2}\left(v_{1}^{\prime}, v_{2}\right)=B_{2}$, so $v \in E_{2}$.

The next lemma further describes the regions $E_{1}$ and $E_{2}$ :

Lemma 5.6 If $P$ is a packing polytope, there is a finite set of vectors $\left\{u^{1}, u^{2}, \ldots, u^{k}\right\}$ such that for $v \neq t u^{i}$ for some $t \geq 0$, the VCG family is uniquely defined. Moreover, if $(x, p)$ is a mechanism with the desirable properties and $E_{1} \cap E_{2} \subseteq\{v ; v \geq a\}$ then if $\xi_{i}\left(v_{-i}\right)=\inf \left\{v_{i} ; p_{i}\left(v_{i}, v_{-i}\right)=B_{i}\right\}$ and $v_{-i}<a_{-i}$, then $\left(\xi_{i}\left(v_{-i}\right), v_{-i}\right)=t u^{i}$ for some $t, i$.

Proof : The set of vectors $\left\{u^{1}, u^{2}, \ldots, u^{k}\right\}$ is simply the set of normals of the edges of the polytope as depicted in the first part of Figure 3 . If $v$ is not an edge in the polytope, then the point in $P$ 

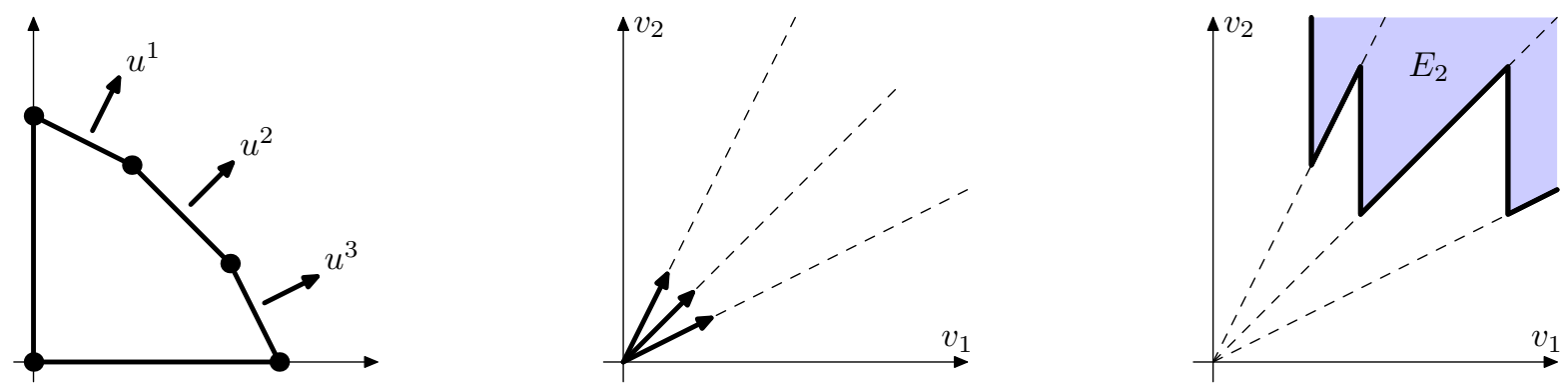

Figure 3: Illustration of the proof of Lemma 5.6

maximizing $v^{t} x$ is a vertex and therefore uniquely defined. If we draw the lines $u^{i} \cdot t$ for $t>0$ we divide the space of all possible valuations in regions (see second part of the figure): the regions correspond to the vertices and the lines to edges of the polytope.

Now, given a certain mechanism $(x, p)$, suppose that $v_{1}<a_{1}$ the vector $\left(v_{1}, \xi_{2}\left(v_{1}\right)\right)$ is not normal to any edge of $P$. Then clearly $x^{*}\left(v_{1}, \xi_{2}\left(v_{1}\right)\right)$ is well-defined and moreover, for some $\delta>0$ and $v_{2}^{\prime} \in\left[\xi_{2}\left(v_{1}\right)-\delta, \xi_{2}\left(v_{1}\right)+\delta\right], x^{*}\left(v_{1}, v_{2}^{\prime}\right)$ is well-defined and constant in the $v_{2}^{\prime}$-range. Also $\left(v_{1}, v_{2}^{\prime}\right) \notin E_{1} \cap E_{2}$, since $v_{1}<a_{1}$. For such $v_{2}^{\prime}>\xi_{2}\left(v_{1}\right)$, we know that $\left(v_{1}, v_{2}^{\prime}\right) \in E_{2}$, thus it is not in $E_{1}$ and therefore $x_{2}\left(v_{1}, v_{2}^{\prime}\right)=x_{2}\left(v_{1}, \xi_{2}\left(v_{1}\right)\right) \leq x_{2}^{*}\left(v_{1}, \xi_{2}\left(v_{1}\right)\right)$. For $v_{2}^{\prime}<\xi_{2}\left(v_{1}\right),\left(v_{1}, v_{2}^{\prime}\right) \notin E_{2}$ so $x_{2}\left(v_{1}, v_{2}^{\prime}\right) \geq x_{2}^{*}\left(v_{1}, v_{2}^{\prime}\right)$. Using that $x_{2}\left(v_{1}, v_{2}^{\prime}\right)$ is constant in $v_{2}^{\prime}$ in this range and taking $v_{2}^{\prime} \uparrow \xi_{2}\left(v_{1}\right)$, we get: $x_{2}\left(v_{1}, v_{2}^{\prime}\right) \geq x_{2}^{*}\left(v_{1}, \xi_{2}\left(v_{1}\right)\right)$.

Now, by monotonicity, we have $x_{2}\left(v_{1}, v_{2}^{\prime}\right)=x_{2}^{*}\left(v_{1}, \xi_{2}\left(v_{1}\right)\right)$ for all $v_{2}^{\prime}$ in the interval $\left(\xi_{2}\left(v_{1}\right)-\right.$ $\left.\delta, \xi_{2}\left(v_{1}\right)+\delta\right)$. Therefore the budget of player 2 could not have been exhausted on $\xi_{2}\left(v_{1}\right)$.

The third part of Figure 3 illustrates how the region $E_{2}$ typically looks like. If $u^{1}, u^{2}, \ldots, u^{k}$ are sorted such that $u^{1}$ corresponds to the edge that is higher to the left and $u^{k}$ corresponds to the edge that is lower to the right, then we can divide $\left[0, a_{1}\right)$ in segments $\left[0, r_{1}\right),\left[r_{1}, r_{2}\right), \ldots,\left[r_{k}, a_{1}\right)$ such that for $v_{1} \in\left[0, r_{1}\right), \xi_{2}\left(v_{1}\right)=\infty$, and for $v_{1} \in\left[r_{i}, r_{i+1}\right),\left(v_{1}, \xi_{2}\left(v_{1}\right)\right)$ is in the line $\left\{t \cdot u^{i} ; t \geq 0\right\}$. To see why this is true, consider $v_{1}<v_{1}^{\prime}$ and assume that $\left(v_{1}, \xi_{2}\left(v_{1}\right)\right)$ is in the line $\left\{t \cdot u^{i} ; t \geq 0\right\}$. Then $\left(v_{1}^{\prime}, \xi_{2}\left(v_{1}^{\prime}\right)\right)$ cannot be strictly above this line, by a similar argument used in Lemma 5.5 look at the allocation curves $x_{2}\left(v_{1}, v_{2}\right) \geq x_{2}\left(v_{1}^{\prime}, v_{2}\right)$ and the payment formula - then player 2 must be paying just above the $\left\{t \cdot u^{i} ; t \geq 0\right\}$ for $v_{1}^{\prime}$ line at least as much as he was paying above this line for $v_{1}$ and hence his budgets must be exhausted.

\subsection{Proof of the Impossibility Theorem}

Now, we are ready to prove Theorem 5.3, which states that there is no general auction with all the desirable properties for all polyhedral environments $P$. We fix the following setting: a set of feasible allocations

$$
P=\left\{x \in \mathbb{R}_{+}^{2} ; 2 x_{1}+x_{2} \leq 6, x_{1}+2 x_{2} \leq 6\right\}
$$

and budgets $B_{1}=B_{2}=1$. Assume that $(x, p)$ is a mechanism with the desirable properties for this setting. We will use the characterization lemmas in Section 5.2 to find a contradiction. We illustrate the flow of the proof in Figure 4 (a brief summary of the proof is given in the caption of the figure).

Fact 5.7 In the region $\left[0, \frac{2}{3}\right) \times\left[0, \frac{2}{3}\right)$, the mechanism $(x, p)$ produces an efficient allocation (i.e., it is equal to some mechanism of the VCG family). 

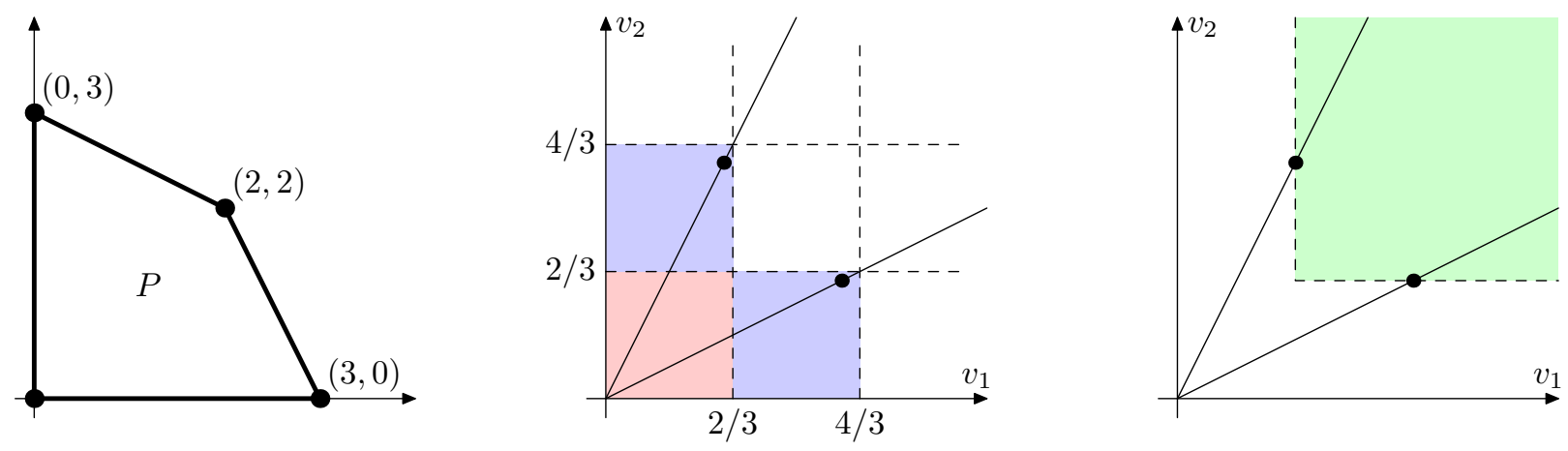

Figure 4: Illustration of the proof of the impossibility theorem: in the first part we represent the polytope $P$, in the second we represent the first main steps: we show that any auction must resemble VCG in the red region (Fact 5.7) and extend the definition of the auction to the blue region (Facts 5.8 and [5.9), showing that the budget of player 1 must get exhausted at the point $\left(\frac{1.2381}{2}, 1.2381\right)$ and the budget of player 2 must get exhausted at the point $\left(1.2381, \frac{1.2381}{2}\right)$. We use this fact to show that the allocation must be constant in the green region (Fact [5.10), contradicting Pareto-optimality for allocation of the form $\left(v_{1}, v_{2}\right)$ where $\frac{1.2381}{2}<v_{1}<\frac{2}{3}$ as $v_{2} \rightarrow \infty$.

Proof : First notice that no mechanism in the VCG-family for $P$ exhausts the budget in $\left[0, \frac{2}{3}\right) \times$ $\left[0, \frac{2}{3}\right)$. Now, we turn our attention to the mechanism $(x, p)$, which is a mechanism with the desirable properties for this setting.

In $\left[0, \frac{1}{3}\right) \times\left[0, \frac{2}{3}\right)$, player 1 cannot exhaust his budget, since $x_{1} \leq 3$ and $v_{1}<\frac{1}{3}$. We claim that in this area the mechanism needs to behave like VCG. If there is a point in this region where $x(v)$ doesn't maximize $v^{t} x$ for $x \in P$, then the budget of player 2 must be exhausted. So, there is some $\left(v_{1}, v_{2}\right)$ such that for $v_{2}^{\prime}>v_{2}, p_{2}\left(v_{1}, v_{2}^{\prime}\right)=B_{2}$ and for $v_{2}^{\prime}<v_{2}, x\left(v_{1}, v_{2}^{\prime}\right)$ is in the VCG family. Now, notice that the allocation for $v_{2}^{\prime}>v_{2}$ must be $x_{2}\left(v_{2}^{\prime}, v_{1}\right) \leq x_{2}^{*}\left(v_{2}^{\prime}, v_{1}\right)$. This contradicts the fact that the budget is exhausted for $v_{2}^{\prime} \downarrow v_{2}$. So, this shows that $(x, p)$ must allocate efficiently on $\left[0, \frac{1}{3}\right) \times\left[0, \frac{2}{3}\right)$. Now, for $\left[0, \frac{2}{3}\right) \times\left[0, \frac{1}{3}\right)$, we can do the same argument. Now, what remains are the points in $\left[\frac{1}{3}, \frac{2}{3}\right) \times\left[\frac{1}{3}, \frac{2}{3}\right)$. Let $v$ be such a point. Notice that for $\left(\frac{1}{3}-\epsilon, v_{2}\right)$ and $\left(v_{1}, \frac{1}{3}-\epsilon\right)$, the allocation must be $(2,2)$ because of the previous argument. By monotonicity, $x(v)=(2,2)$ which is the efficient allocation.

Now, we know how any mechanism $(x, p)$ with the desirable properties should look like in the red region of Figure 4. Next, we try to understand how it should look in the blue region. In order to do so, we need some definitions. From Lemma 5.5 we know that there is $\left.a \in \mathbb{R}_{+}^{2} \cup\{(\infty, \infty)\}\right)$ such that: $\{v ; v>a\} \subseteq E_{1} \cap E_{2} \subseteq\{v ; v \geq a\}$. We also define:

$$
\begin{aligned}
& \tilde{v}_{2}=\min \left\{v_{2} ; p_{1}\left(\frac{v_{2}}{2}+, v_{2}\right)=1\right\} \\
& \tilde{v}_{1}=\min \left\{v_{1} ; p_{2}\left(v_{1}, \frac{v_{1}}{2}+\right)=1\right\}
\end{aligned}
$$

And we focus on the region $R$, which we define as the interior of the rectangle between $(0,0)$ and $\left(\tilde{v}_{2} / 2, \tilde{v}_{2}\right)$.

Fact 5.8 The budget of player 1 does not get exhausted in $R$. Also, $\frac{2}{3}<\tilde{v}_{2} \leq 1.2381$

Proof : First, assume $\tilde{v}_{2}<4 / 3$. Then by Fact [5.7, the budget of player 1 does not get exhausted in $\left[0, \frac{2}{3}\right) \times\left[0, \frac{2}{3}\right)$ and by the definition of $\tilde{v}_{2}$ and lemma 5.6] it cannot be exhausted for $\left[0, \frac{\tilde{v}_{2}}{2}\right) \times\left(\frac{1}{3}, \tilde{v}_{2}\right)$. Notice that $a$ cannot be in this region, because it would also contradict the definition of $\tilde{v}_{2}$. 
Given this fact, let us analyze how the mechanism should be in this setting. We do so, by fixing $v_{1}$ and looking at $x_{2}\left(v_{1}, v_{2}\right)$. We can use the same argument as in the previous fact to argue that $x\left(v_{1}, v_{2}\right)$ must be the efficient allocation for $v_{2}<2 v_{1}$ in $R$ and also for all $v_{1} \leq \frac{1}{3}$. For $v_{1}>\frac{1}{3}$ and $v_{2}>2 v_{1}$, if we allocate as in VCG, we exceed the budget. So, the allocation for those points must exactly match the budget of player 2 . So, the only possible value must be such that:

$$
2 \cdot \frac{1}{2} v_{1}+\left(x_{2}(v)-2\right) 2 v_{1}=1
$$

and therefore the allocation must be

$$
x(v)=\left(3-\frac{1}{v_{1}}, 2+\frac{1-v_{1}}{2 v_{1}}\right) .
$$

Now, this determines the payment of player 1 in the rectangle. We know that for $\delta \downarrow 0$, $p_{1}\left(\frac{\tilde{v}_{2}}{2}-\delta, \tilde{v}_{2}-3 \delta\right)<1$, which we can write as:

$$
\lim _{\delta \downarrow 0} p_{1}\left(\frac{\tilde{v}_{2}}{2}-\delta, \tilde{v}_{2}-3 \delta\right)=2 \cdot \frac{\tilde{v}_{2}}{2}-\int_{1 / 3}^{\tilde{v}_{2} / 2} 3-\frac{1}{z} d z=1-\frac{\tilde{v}_{2}}{2}+\log \left(\frac{3}{2} \tilde{v}_{2}\right) \leq 1
$$

This implies that $\tilde{v}_{2} \leq 1.2381$. Notice that this excludes the fact that $\tilde{v}_{2}>4 / 3$, otherwise we could have done the same analysis on $\left[0, \frac{2}{3}\right] \times\left[0, \tilde{v}_{2}\right]$ and arrived in the same conclusion that $\tilde{v}_{2} \leq 1.2381$.

Fact $5.9 \tilde{v}_{2}=1.2381$ and $x\left(\frac{\tilde{v}_{2}}{2}+, \tilde{v}_{2}\right)=(2,2)$.

Proof : Since $\frac{2}{3}<\tilde{v}_{2} \leq 1.2381$, we know that $x_{2}\left(\frac{\tilde{v}_{2}}{2}+, \tilde{v}_{2}\right) \geq 2$ by using that $x_{2}\left(\frac{\tilde{v}_{2}}{2}+, \frac{1}{3}\right)=2$ (Fact 5.7) and monotonicity. Therefore, $x_{1}\left(\frac{\tilde{v}_{2}}{2}+, \tilde{v}_{2}\right) \leq 2$. Writing the payment for player 1 at this point we get:

$$
1=p_{1}\left(\frac{\tilde{v}_{2}}{2}+, \tilde{v}_{2}\right)=x_{1}\left(\frac{\tilde{v}_{2}}{2}+, \tilde{v}_{2}\right) \cdot \frac{\tilde{v}_{2}}{2}-\int_{1 / 3}^{\tilde{v}_{2} / 2} 3-\frac{1}{z} d z \leq 1-\frac{\tilde{v}_{2}}{2}+\log \left(\frac{3}{2} \tilde{v}_{2}\right)
$$

which implies that $\tilde{v}_{2}=1.2381$ and $x\left(\frac{\tilde{v}_{2}}{2}+, \tilde{v}_{2}\right)=(2,2)$, since all inequalities must be tight.

Fact $5.10 \tilde{v}_{1}=\tilde{v}_{2}=1.2381$ and $x(v)=(2,2)$ for all valuation profiles $v>\left(\frac{\tilde{v}_{1}}{2}, \frac{\tilde{v}_{2}}{2}\right)$.

Proof : We can apply the same argument exchanging 1 and 2 and conclude that $\tilde{v}_{1}=\tilde{v}_{2}$. Now, to see that for $v_{1}^{\prime}, v_{2}^{\prime}>\frac{\tilde{v}_{i}}{2}$ we have $x\left(v_{1}^{\prime}, \tilde{v}_{2}\right)=x\left(\tilde{v}_{1}, v_{2}^{\prime}\right)=(2,2)$, we analyze four regions. If $v \in\left(\tilde{v}_{i} / 2, \tilde{v}_{i}\right] \times\left(\tilde{v}_{i} / 2, \tilde{v}_{i}\right]$ or $v \in\left[\tilde{v}_{i}, \infty\right) \times\left[\tilde{v}_{i}, \infty\right)$ we can use the standard monotonicity argument to show that $x(v)=(2,2)$.

For the regions $\left[\tilde{v}_{1}, \infty\right) \times\left(\frac{\tilde{v}_{1}}{2}, \tilde{v}_{1}\right)$ and $\left(\frac{\tilde{v}_{2}}{2}, \tilde{v}_{2}\right) \times\left[\tilde{v}_{2}, \infty\right)$ is a little trickier. We do the analysis for the first one. The second is analogous.

Clearly $p_{2}\left(\tilde{v}_{1}, \tilde{v}_{2}\right)=1$. Now, for $v_{1}^{\prime}>\tilde{v}_{1}, x\left(v_{1}^{\prime}, \tilde{v}_{2}\right)=x\left(\tilde{v}_{1}, \tilde{v}_{2}\right)=(2,2)$. And for all $v_{2}$ we have $x_{1}\left(v_{1}^{\prime}, v_{2}\right) \geq x_{1}\left(\tilde{v}_{1}, v_{2}\right)$ and therefore $x_{2}\left(v_{1}^{\prime}, v_{2}\right) \leq x_{2}\left(\tilde{v}_{i}, v_{2}\right)$. Now, since $p_{2}\left(v_{1}, v_{2}\right)=v_{2} x_{2}(v)-$ $\int_{0}^{v_{2}} x_{2}\left(v_{1}, u\right) d u$ clearly $p_{2}\left(v_{1}^{\prime}, \tilde{v}_{2}\right) \geq p_{2}\left(\tilde{v}_{1}, \tilde{v}_{2}\right)=1$. Therefore $p_{2}\left(v_{1}^{\prime}, \tilde{v}_{2}\right)=1$, since the mechanism respects budgets. Notice that the only way it can be true is that if $x_{2}\left(v_{1}^{\prime}, v_{2}\right)=x_{2}\left(\tilde{v}_{i}, v_{2}\right)$, so we must have $x_{2}\left(v_{1}^{\prime}, v_{2}\right)=(2,2)$ for $v_{1}^{\prime} \in\left[\tilde{v}_{i}, \infty\right)$.

We can use the exact same argument for region: $\left(\frac{\tilde{v}_{i}}{2}, \tilde{v}_{i}\right) \times\left[\tilde{v}_{i}, \infty\right)$. 
Now we are ready to prove Theorem 5.3 .

Proof of Theorem 5.3: Now, by putting Fact 5.10 and Fact 5.7 together, we get a contradiction with the Pareto-optimality: consider $\frac{\tilde{v}_{i}}{2}<v_{1}<\frac{2}{3}$ then by facts 5.10 and 5.7 combined, we know that $x_{2}\left(v_{1}, v_{2}\right)=0$ for $v_{2}<\frac{v_{1}}{2}$ and $x_{2}\left(v_{1}, v_{2}\right)=2$ for $v_{2}>\frac{v_{1}}{2}$, so the budget of player 2 never gets exhausted even for $v_{2} \rightarrow \infty$. This contradicts Pareto-optimality for $v_{2}>2 v_{1}$, since if his budget is not exhausted, he should get allocated at least as much as he gets in VCG.

\subsection{Multi-unit auctions with decreasing marginals}

As a by-product of Theorem 5.3, we can answer in a negative way the question of the existence of truthful Pareto-optimal auctions for multi-unit auctions with decreasing marginals. Consider the following setting:

Setting: Consider a supply of $s$ of a certain divisible good and two players in such a way that the feasible allocations are $\left(x_{1}, x_{2}\right)$ such that $x_{1}+x_{2} \leq s$. Player $i$ has a public budget $B_{i}$ and a private valuation which is a increasing concave function $V_{i}:[0, s] \rightarrow \mathbb{R}_{+}$. Upon getting $x_{i}$ units of the good and paying $p_{i}$, player $i$ has utility $u_{i}=V_{i}\left(x_{i}\right)-p_{i}$.

It is tempting to believe that one could adapt the clinching framework in Algorithm 1 to deal with this setting, by simply redefining the demand function as something like:

$$
d_{i}=\min \left\{\frac{B_{i}}{p}, \max \left\{x_{i} ; \partial V_{i}\left(\rho_{i}+x_{i}\right) \leq p\right\}\right\}
$$

where $\partial V_{i}\left(x_{i}\right)$ is the marginal valuation at $x_{i}$. Indeed, if $V_{i}\left(x_{i}\right)=v_{i} \cdot x_{i}$, this recovers the original way of calculating demands. In appendix $\mathrm{D}$ we give a counter-example showing that the clinching framework with this new demand function results in a non-truthful mechanism. The intuition behind the counter example is that some player can increase his declared value on items he won't get anyway in order to increase the payment of his opponent, exhausting his budget earlier. This way, he is able to get items for cheaper in the end.

In the following theorem, we show that no auction mechanism can satisfy all the desirable properties for this setting:

Theorem 5.11 There is no truthful, Pareto-optimal and budget-feasible auction for this setting.

Proof : Suppose that $\left(\hat{x}\left(V_{1}, V_{2}\right), \hat{p}\left(V_{1}, V_{2}\right)\right)$ is a mechanism satisfying all the desirable properties for multi-unit auctions with decreasing marginals. Then we can use it as a black-box to construct a mechanism for a general polyhedral environment, contradicting Theorem 5.3. Given a certain polyhedral environment, we can describe

$$
P=\left\{x \in[0, \alpha] \times[0, \beta] ; x_{2} \leq h\left(x_{1}\right)\right\}
$$

where $h:[0, \alpha] \rightarrow[0, \beta]$ is a monotone non-increasing concave function, $h(0)=\beta$ and $h(\alpha)=0$. Now, using $(\hat{x}, \hat{p})$ for $s=1$, build the following mechanism: if players report valuations $v_{1}, v_{2}$ build the following concave functions: $V_{1}\left(x_{1}\right)=v_{1} \cdot \alpha x_{1}$ and $V_{2}\left(x_{2}\right)=v_{2} \cdot h\left(\alpha-\alpha x_{2}\right)$.

Now, simply define

$$
\begin{aligned}
& x\left(v_{1}, v_{2}\right)=\left(\alpha \hat{x}_{1}, h\left(\alpha \hat{x}_{1}\right)\right) \\
& p\left(v_{1}, v_{2}\right)=\hat{p}\left(V_{1}, V_{2}\right) .
\end{aligned}
$$


The mechanism is clearly truthful, individually rational and budget-feasible. It is also easy to see that sets of allocation can me mapped 1-1 between those two settings, preserving Pareto-optimality.

\section{References}

[1] Z. Abrams. Revenue maximization when bidders have budgets. In SODA, pages 1074-1082, 2006.

[2] G. Aggarwal, S. Muthukrishnan, D. Pál, and M. Pál. General auction mechanism for search advertising. In $W W W$, pages $241-250,2009$.

[3] A. Archer and É. Tardos. Truthful mechanisms for one-parameter agents. In FOCS, pages 482-491, 2001.

[4] I. Ashlagi, M. Braverman, A. Hassidim, R. Lavi, and M. Tennenholt. Position auctions with budgets: Existence and uniqueness. In B.E. journal of Theoretical Economics Advances, forthcoming, 2011.

[5] L. M. Ausubel. An efficient ascending-bid auction for multiple objects. American Economic Review, 94, 1997.

[6] J.-P. Benoit and V. Krishna. Multiple-object auctions with budget constrained bidders. Review of Economic Studies, 68(1):155-79, January 2001.

[7] S. Bhattacharya, V. Conitzer, K. Munagala, and L. Xia. Incentive compatible budget elicitation in multi-unit auctions. In SODA, pages 554-572, 2010.

[8] S. Bikhchandani, S. de Vries, J. Schummer, and R. V. Vohra. An ascending vickrey auction for selling bases of a matroid. Operations Research, 59(2):400-413, 2011.

[9] C. Borgs, J. T. Chayes, N. Immorlica, M. Mahdian, and A. Saberi. Multi-unit auctions with budget-constrained bidders. In ACM Conference on Electronic Commerce, pages 44-51, 2005.

[10] I. Caragiannis, P. Kanellopoulos, C. Kaklamanis, and M. Kyropoulou. On the efficiency of equilibria in generalized second price auctions. In $E C^{\prime} 11,2011$.

[11] Y.-K. Che and I. Gale. Standard auctions with financially constrained bidders. Review of Economic Studies, 65(1):1-21, January 1998.

[12] R. Colini-Baldeschi, M. Henzinger, S. Leonardi, and M. Starnberger. On multiple round sponsored search auctions with budgets. CoRR, abs/1112.6361, 2011.

[13] P. Dhangwatnotai. Multi-keyword sponsored search. In ACM Conference on Electronic Commerce, pages 91-100, 2011.

[14] S. Dobzinski, R. Lavi, and N. Nisan. Multi-unit auctions with budget limits. In FOCS, pages 260-269, 2008.

[15] D. Dolev, D. G. Feitelson, J. Y. Halpern, R. Kupferman, and N. Linial. No justified complaints: on fair sharing of multiple resources. In ICTS, pages 68-75, 2012. 
[16] B. Edelman, M. Ostrovsky, and M. Schwarz. Internet advertising and the generalized secondprice auction: Selling billions of dollars worth of keywords. The American Economic Review, 97(1):242-259, March 2007.

[17] J. Feldman, S. Muthukrishnan, E. Nikolova, and M. Pál. A truthful mechanism for offline ad slot scheduling. In SAGT, pages 182-193, 2008.

[18] A. Fiat, S. Leonardi, J. Saia, and P. Sankowski. Single valued combinatorial auctions with budgets. In ACM Conference on Electronic Commerce, pages 223-232, 2011.

[19] R. L. Graham, E. L. Lawler, J. K. Lenstra, and A. H. G. R. Kan. Optimization and approximation in deterministic sequencing and scheduling: a survey. Ann. Discrete Math., 4:287-326, 1979 .

[20] I. Hafalir, R. Ravi, and A. Sayedi. Sort-cut: A pareto optimal and semi-truthful mechanism for multi-unit auctions with budget-constrained bidders. Ad Auctions Workshop.

[21] R. Lavi and M. May. A note on the incompatibility of strategy-proofness and pareto-optimality in quasi-linear settings with public budgets. WINE.

[22] B. Lucier and R. Paes Leme. Gsp auctions with correlated types. In EC'11, 2011.

[23] B. Lucier, R. Paes Leme, and Éva Tardos. On revenue in the generalized second price auction. In Ad Auctions Workshop, 2011.

[24] C. J. H. McDiarmid. Rado's theorem for polymatroids. Mathematical Proceedings of the Cambridge Philosophical Society, 78(02):263-281, 1975.

[25] R. Myerson. Optimal auction design. Mathematics of Operations Research, 6(1):58-73, 1981.

[26] T. Nguyen and É. Tardos. Approximately maximizing efficiency and revenue in polyhedral environments. In ACM Conference on Electronic Commerce, pages 11-19, 2007.

[27] T. Nguyen and M. Vojnovic. The weighted proportional sharing mechanisms. In SIGMETRICS, 2011.

[28] R. Paes Leme and E. Tardos. Pure and bayes-nash price of anarchy for generalized second price auctions. In FOCS10: 51st Annual IEEE Symposium on Foundations of Computer Science, 2010 .

[29] M. M. Pai and R. Vohra. Optimal auctions with financially constrained bidders. Discussion papers, Northwestern University, Center for Mathematical Studies in Economics and Management Science, Aug 2008.

[30] A. Schrijver. Combinatorial Optimization - Polyhedra and Efficiency. Springer, 2003.

[31] H. R. Varian. Position auctions. International Journal of Industrial Organization, 2006. 


\section{A Extensions and Limitations of the clinching framework}

Scaled polymatroids: If $P \subseteq \mathbb{R}_{+}^{n}$ is a polymatroid, $\gamma \in \mathbb{R}_{+}^{n}$, then we call $P_{\gamma}=\left\{x ;\left(\frac{x_{i}}{\gamma_{i}}\right)_{i} \in P\right\}$ a scaled polymatroid. If the environment is $P_{\gamma}$, it is easy to see that a truthful, individually-rational, Pareto-optimal and budget-feasible auction is obtained by running the polymatroid clinching auction on $P$ with inputs $\gamma_{i} v_{i}$ instead of $v_{i}$. It is simple to see that this is equivalent to a standard clinching auction with input values $v_{i}$ but price clocks advancing on a different speed for each player. Scaled polymatroids are important since they correspond to the setting of AdWords with Quality Factors discussed at the end of section 4.1).

Beyond scaled polymatroids: one could change the way clinching is done and one could change the price trajectories, maybe in a more sophisticated way then the one we did for the scaled polymatroids. If the trajectory is such that it only depends on $P$ and budgets (not on values) and $p_{i}$ never decreases, the auction retains truthfulness and budget feasibility. Here we argue that none of such changes would generate a Pareto-optimal auction when $P$ is not a scaled polymatroid. Assume $n=2$ for simplicity and imagine that there is trajectory for the price vector $p$ and a clinching procedure. Also assumes valuations are much smaller than budgets in such a way that the mechanism cannott exhaust budgets and therefore the auction must allocate like VCG. Any such mechanism must decide on the whole allocation the first time $p_{i}=v_{i}$ for some component, having only the information that $v_{j} \geq p_{j}$ for the other component, since at this point he needs to allocate to the second player. So, the environment must be such that there is a price trajectory $p(t)$, where the optimal allocation is constant for all points $\left(p_{1}, v_{2}\right)$ for all $v_{2}>p_{2}$. And also, it should be constant for all $\left(v_{1}, p_{2}\right)$ for all $v_{1}>p_{1}$. Notice that scaled polymatroids are exactly those environments.

\section{B Faster clinching sub-routines}

In Lemma 3.5 we showed that for any generic polymatroidal environment $P$ we can perform the clinching step in polynomial time if we have oracle access to the submodular function defining the polymatroid. In order to do so, we solve a submodular minimization problem. For most practical applications, however, one can design much simpler and faster algorithms for clinching. Clinching involves solving the following problem: given an environment $P, \rho \in P$ and $d \in \mathbb{R}_{+}^{n}$ we want to compute:

$$
\max \left\{\mathbb{1}^{t} x ; x+\rho \in P, 0 \leq x \leq d\right\}
$$

We illustrate how to solve this problem efficiently for the single-keyword AdWords polytope: given $\alpha_{1} \geq \alpha_{2} \geq \ldots \geq \alpha_{n}$, consider the environment:

$$
P=\left\{x \in \mathbb{R}_{+}^{n} ; x(S) \leq \sum_{j=1}^{|S|} \alpha_{j}, \forall S \subseteq[n]\right\}
$$

Lemma B.1 For the single-keyword AdWords polytope, the optimization problem defined in equation (3) can be solved using the following greedy algorithm: we can assume wlog that the components are sorted such that $\rho_{1}+d_{1} \geq \rho_{2}+d_{2} \geq \ldots \geq \rho_{n}+d_{n}$. Now, define inductively

$$
z_{i}=\min \left\{\rho_{i}+d_{i}, \sum_{j=1}^{i} \alpha_{j}-\sum_{j=1}^{i-1} z_{j}\right\} .
$$

Then $\sum_{i} z_{i}-\rho_{i}$ is the solution to the problem. 
Proof : If we drop the restriction that $x \geq 0$ in (3), then it is easy to see that $x=z-\rho$ is an optimal solution to this problem using, for example, a local exchange argument. Now, we show that we can fix this problem, by modifying $z$ such that $z \geq \rho$.

In order to fix that, consider the smaller $i$ such that $z_{i}<\rho_{i}$. By the definition of $z_{i}$, it must be the case that $z_{i}=\sum_{j=1}^{i} \alpha_{j}-\sum_{j=1}^{i-1} z_{j}$, so $\sum_{j=1}^{i} z_{j}=\sum_{j=1}^{i} \alpha_{j}$. Then there must be some $k<i$ such that $z_{k}>\rho_{k}$, otherwise we would have $\sum_{j=1}^{i} \rho_{j}>\sum_{j=1}^{i} \alpha_{j}$ contradicting the fact that $\rho \in P$. Notice we can increase $z_{i}$ by some small $\delta$ and decrease $z_{k}$ by a small $\delta$. And obtain another vector $z$ which is also such that $z \in P, z \leq \rho+d$ and has the same $\mathbb{1}^{t} z$ value (the fact that $z \in P$ after this transformation is due to the nature of the constraints). We can repeat this process until we get $z \geq \rho$.

\section{Missing proofs in Section 5}

Proof of Lemma 5.4: $\quad$ Suppose that $x\left(v_{1}+, v_{2}\right) \neq \tilde{x}\left(v_{1}+, v_{2}\right)$, then say that $x_{1}\left(v_{1}+, v_{2}\right)>$ $\tilde{x}_{1}\left(v_{1}+, v_{2}\right)$. Since both are in the boundary of the polytope, it means that for all $\left(v_{1}^{\prime}, v_{2}\right)$ with $v_{1}^{\prime}>v_{1}, \tilde{x}_{1}\left(v_{1}^{\prime}, v_{2}\right) \geq x_{1}\left(v_{1}+, v_{2}\right)$, since the points to the right of $x\left(v_{1}+, v_{2}\right)$ are clearly better then the ones to the left of it. So $\tilde{x}_{1}\left(v_{1}^{\prime}, v_{2}\right)$ can't converge to $\tilde{x}_{1}\left(v_{1}+, v_{2}\right)<x_{1}\left(v_{1}, x_{2}\right)$.

\section{Counter-example to clinching with decreasing marginals}

Consider the setting of multi-unit auctions with decreasing marginals described in section [5.4, and the variant of the clinching auction in Algorithm 1 where demands are calculated by:

$$
d_{i}=\min \left\{\frac{B_{i}}{p}, \max \left\{x_{i} ; \partial V_{i}\left(\rho_{i}+x_{i}\right) \leq p\right\}\right\}
$$

Our main claim here is that this auction is not truthful. We show that by providing an example: consider an initial supply of $s=2$ budgets $B_{1}=\infty, B_{2}=4$ and valuations:

$$
V_{1}(x)=\left\{\begin{array}{rl}
4 x, & x \in[0,1] \\
4+x, & x \in[1,2]
\end{array} \quad V_{2}(x)=3 x, \quad x \in[0,2]\right.
$$

The outcome of that is $x_{1}=1, x_{2}=1$ and $p_{1}=3, p_{2}=1$ since budgets never get exhausted. Now, we show that player 1 has a profitable deviation. He could report the following valuation instead:

$$
\tilde{V}_{1}(x)=\left\{\begin{aligned}
4 x, & x \in[0,1] \\
4+2 x, & x \in[1,2]
\end{aligned}\right.
$$

The the price clock increases up to 2 without any clinching. For $2+$, the demand of player 1 drops and player 2 is able to clinch one unit by the price of 2 , remaining with budget 2 . Now, as price goes up, player 2's demand keeps decreasing, since his leftover budget is only 2 and the price is greater than 2 . As a result, player 1 will be able to start clinching at price $p=2+$ and therefore will have $\tilde{x}_{1}=1, \tilde{p}_{1}<3$, which is a strict improvement over the truth-telling strategy. 